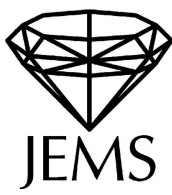

Camillo De Lellis · Felix Otto

\title{
Structure of entropy solutions to the eikonal equation
}

Received June 24, 2002 / final version received November 12, 2002

Published online February 7, 2003 - (c) Springer-Verlag \& EMS 2003

Abstract. In this paper, we establish rectifiability of the jump set of an $\mathbf{S}^{1}$-valued conservation law in two space-dimensions. This conservation law is a reformulation of the eikonal equation and is motivated by the singular limit of a class of variational problems. The only assumption on the weak solutions is that the entropy productions are (signed) Radon measures, an assumption which is justified by the variational origin. The methods are a combination of Geometric Measure Theory and elementary geometric arguments used to classify blow-ups.

The merit of our approach is that we obtain the structure as if the solutions were in $\mathrm{BV}$, without using the BV-control, which is not available in these variationally motivated problems.

Key words. entropy solutions - partial regularity - singular perturbation - conservation laws - rectifiability

\section{Introduction}

\subsection{Motivation}

Consider an energy functional of the form

$$
F_{\varepsilon}\left(m_{\varepsilon}\right)=\int_{\Omega}\left(\varepsilon\left|\nabla m_{\varepsilon}\right|^{2}+\frac{1}{\varepsilon}\left(1-\left|m_{\varepsilon}\right|^{2}\right)^{2}\right)+\frac{1}{\varepsilon} \int_{\mathbf{R}^{2}}\left|\nabla^{-1}\left(\nabla \cdot m_{\varepsilon}\right)\right|^{2}
$$

defined on the space of vector fields $m: \Omega \rightarrow \mathbf{R}^{2}$. Here

$$
\int_{\mathbf{R}^{2}}\left|\nabla^{-1}\left(\nabla \cdot m_{\varepsilon}\right)\right|^{2}=\int_{\mathbf{R}^{2}}|\nabla u|^{2} \quad \text { where }-\Delta u=\nabla \cdot m_{\varepsilon} .
$$

In this paper, we study the regularity of elements $m$ of the "asymptotic admissible set". By the asymptotic admissible set of a sequence of functionals $\left\{F_{\varepsilon}\right\}_{\mathcal{E} \downarrow 0}$, we

C. De Lellis: Max-Planck Institute for Mathematics in the Sciences, Inselstr. 22-26, 04103 Leipzig, Germany, e-mail: delellis@ @mis.mpg.de

F. Otto: Institut für Angewandte Mathematik, University of Bonn, Wegelerstr. 10, 53115 Bonn, Germany, e-mail: otto@iam.uni-bonn.de 
understand the set of all strong limits $m$ (say, in $L^{p}(\Omega)$ for all $p<\infty$ ) of sequences $\left\{m_{\varepsilon_{n}}\right\}_{n \uparrow \infty}$ which are bounded in energy.

What can we expect? In view of the $\frac{1}{\varepsilon}$-terms in (1), such a limit $m$ satisfies

$$
|m|^{2}=1 \text { a.e. and } \nabla \cdot m=0 \text { distributionally. }
$$

There are two ways of looking upon (2) which are particular to two space dimensions. The first point of view is: since $\nabla \cdot m=0$, there exists a stream function $\psi$ such that $m$ can be written as its gradient rotated by $\frac{\pi}{2}$, that is, ${ }^{\perp} \nabla \psi=m$. Then the first condition of (2) turns into the eikonal equation

$$
|\nabla \psi|^{2}=1 \text { a.e. . }
$$

The second point of view is: since $|m|^{2}=1$, we may introduce a phase $\theta$ such that $m$ can be written as $\left(m_{1}, m_{2}\right)=(\cos \theta, \sin \theta)$. Then the second condition of (2) turns into a scalar conservation law

$$
\frac{\partial \cos \theta}{\partial x_{1}}+\frac{\partial \sin \theta}{\partial x_{2}}=0 \text { distributionally. }
$$

Both (3) and (4) are rigid for smooth $\psi$ resp. $\theta$, as can be seen from the characteristics of these first order equations. But they practically lose all this rigidity if $\psi$ is only Lipschitz or $\theta$ is only an essentially bounded function. The concept of viscosity solution resp. of entropy solution restores the "right amount" of rigidity. But these concepts seem a priori unrelated to our variational problem.

Which properties beyond (2) can be expected? In view of the $\varepsilon$ in front of the Dirichlet integral, finite-energy limits $m$ will not be smooth in general. As we shall presently see, the scaling of the energy $F_{\varepsilon}$ is just such that it "sees" one-dimensional discontinuities (jumps) of the limit $m$. In view of (2), the normal component of $m$ is continuous across jumps. The line-energy density associated with jumps of $m$ can be inferred from the one-dimensional version of (1), the local variational problem

$$
\tilde{F}_{\varepsilon}\left(m_{\varepsilon}\right):=\int\left(\varepsilon\left|\frac{d m_{\varepsilon}}{d x_{1}}\right|^{2}+\frac{1}{\varepsilon}\left(1-\left|m_{\varepsilon}\right|^{2}\right)^{2}+\frac{1}{\varepsilon}\left(m_{\varepsilon, 1}-m_{0,1}\right)^{2}\right) d x_{1} .
$$

Here $m_{0,1}$ corresponds to the (prescribed) normal component of $m$. Rescaling length as $x_{1}=\varepsilon \hat{x}_{1}$, one sees that the line-energy density is indeed $O(1)$ in $\varepsilon$. More precisely, a standard calculation shows that for $m_{0}^{ \pm} \in \mathbf{S}^{1}$ with $m_{0,1}^{ \pm}=m_{0,1}$,

$$
\min \left\{\tilde{F}_{\varepsilon}\left(m_{\varepsilon}\right) \mid m_{\varepsilon} \rightarrow m_{0}^{ \pm} \text {as } x_{1} \rightarrow \pm \infty\right\}=O\left(\left|m_{0}^{+}-m_{0}^{-}\right|^{3}\right) .
$$

The fact that the line-energy density is $O(1)$ in $\varepsilon$ naively suggests that a finiteenergy limit $m$ has a moderately regular one-dimensional discontinuity set. The cubic degeneracy $O\left(\left|m_{0}^{+}-m_{0}^{-}\right|^{3}\right)$ in the jump size $\left|m_{0}^{+}-m_{0}^{-}\right|$on the other hand indicates that we possibly do not control the total variation of $m$ (more discussion on this in Subsect. 1.4). In the main result of this paper, Theorem 1, we will nevertheless establish regularity properties for $m$ as if it were of bounded variation. 


\subsection{Statement of result}

The point of view (4) suggests to borrow the concept of entropies from conservation laws to further characterize the asymptotic admissible set. Following [13], we introduce

Definition 1. A smooth and compactly supported function $\Phi: \mathbf{R}^{2} \rightarrow \mathbf{R}^{2}$ will be called an entropy if for every open set $\Omega$ and every smooth $m: \Omega \rightarrow \mathbf{R}^{2}$ we have

$$
\left(\nabla \cdot m=0 \text { and }|m|^{2}=1\right) \Longrightarrow \nabla \cdot[\Phi(m)]=0 .
$$

A particular set of entropies has first been introduced by Jin and Kohn as "calibrations" to establish lower bounds on the energy which are optimal in the limit $\varepsilon \downarrow 0$ [19]. Later, the concept of entropies, together with other tools from conservation laws such as the div-curl-Lemma and Young measures, has been used to establish

$$
\left\{F_{\varepsilon}\left(m_{\varepsilon}\right)\right\}_{\varepsilon \downarrow 0} \text { bounded } \Longrightarrow\left\{m_{\varepsilon}\right\}_{\varepsilon \downarrow 0} \subset L^{p} \text { precompact, }
$$

see [2], [13], [26]. An important ingredient was the estimate

$$
\left|\int \nabla \cdot\left[\Phi\left(m_{\varepsilon}\right)\right] \zeta\right| \leq C_{\Phi}\left(F_{\varepsilon}\left(m_{\varepsilon}\right) \sup |\zeta|+\left(\varepsilon F_{\varepsilon}\left(m_{\varepsilon}\right) \int|\nabla \zeta|^{2}\right)^{1 / 2}\right)
$$

for an arbitrary entropy $\Phi$ and test function $\zeta$. As a variation of Definition 1.3 in [2], this motivates the following:

Definition 2. We call $A(\Omega)$ the set of essentially bounded $m: \Omega \rightarrow \mathbf{R}^{2}$ with (2) and such that for every entropy $\Phi$,

$$
\mu_{\Phi}:=\nabla \cdot[\Phi(m)] \quad \text { is a measure of locally finite total variation. }
$$

We call the $\mu_{\Phi}$ 's entropy measures.

In view of (7), the asymptotic admissible set is a subset of $A(\Omega)$. Our main result is on the structure of $m \in A(\Omega)$ :

Theorem 1. For $m \in A(\Omega)$ there exists $J \subset \Omega$ such that

(a) $J$ is $\mathcal{H}^{1} \sigma$-finite and rectifiable;

(b) for $\mathcal{H}^{1}$-a.e. $x \notin J$,

$$
\lim _{r \downarrow 0} \frac{1}{r^{2}} \int_{B_{r}(x)}\left|m(y)-\bar{m}_{x, r}\right| d y=0,
$$

where $\bar{m}_{x, r}$ is the average of $m$ on $B_{r}(x)$;

(c) for $\mathcal{H}^{1}$-a.e. $x \in J$, there exist $m^{+}(x), m^{-}(x) \in \mathbf{S}^{1}$ with

$$
\lim _{r \downarrow 0} \frac{1}{r^{2}}\left\{\int_{B_{r}^{+}(x)}\left|m(y)-m^{+}(x)\right| d y+\int_{B_{r}^{-}(x)}\left|m(y)-m^{-}(x)\right| d y\right\}=0,
$$

where $B_{r}^{ \pm}(x):=\left\{y \in B_{r}(x) \mid \pm y \cdot \eta(x)>0\right\}$ and $\eta(x)$ is a unit vector normal to $J$ in $x$; 
(d) for every entropy $\Phi$

$$
\begin{aligned}
\mu_{\Phi}\llcorner J & =\left[\eta \cdot\left(\Phi\left(m^{+}\right)-\Phi\left(m^{-}\right)\right)\right] \mathcal{H}^{1}\llcorner J \\
\mu_{\Phi}\llcorner K & =0 \quad \text { for any } K \subset \Omega \backslash J \text { with } \mathcal{H}^{1}(K)<\infty .
\end{aligned}
$$

This is somewhat less than what we would get for free if $m$ had bounded total variation using the fine properties of BV functions and the Vol'pert Chain Rule (see Sect. 3.7 and Theorem 3.96 of [3]). Despite the fact that we cannot expect bounded total variation, we conjecture that $m$ has the same structure. Hence we expect that points (b) and (d) can be improved to

\section{Conjecture 1.}

(b') for $\mathcal{H}^{1}$-a.e. $x \notin J, x$ is a Lebesgue point of $m$.

(d') $\mu_{\Phi}=\left[\eta \cdot\left(\Phi\left(m^{+}\right)-\Phi\left(m^{-}\right)\right)\right] \mathcal{H}^{1}\llcorner J$ for every entropy $\Phi$.

\subsection{Mathematical context}

Why are we interested in (1)? Because its asymptotic admissible set contains the asymptotic admissible sets for two other problems which have been intensively studied in the past years:

Problem 1. The functionals

$$
F_{\varepsilon}^{1}\left(m_{\varepsilon}\right)=\int_{\Omega}\left(\varepsilon\left|\nabla m_{\varepsilon}\right|^{2}+\frac{1}{\varepsilon}\left(1-\left|m_{\varepsilon}\right|^{2}\right)^{2}\right)
$$

on the set of vector fields satisfying $\nabla \cdot m_{\varepsilon}=0$.

Problem 2. The functionals

$$
F_{\varepsilon}^{2}\left(m_{\varepsilon}\right)=\int_{\Omega} \varepsilon\left|\nabla m_{\varepsilon}\right|^{2}+\frac{1}{\varepsilon} \int_{\mathbf{R}^{2}}\left|\nabla^{-1}\left(\nabla \cdot m_{\varepsilon}\right)\right|^{2}
$$

on the set of vector fields satisfying $\left|m_{\varepsilon}\right|^{2}=1$.

Problem 1 was first considered by Aviles and Giga [6]. It was later proposed by Gioia and Ortiz [24] as a model for delamination of compressed thin elastic films ("blisters"), where the stream function $\psi$ is the height of the delamination (for more on modeling of thin-film blistering phenomena see [8]). Since then, many partial results on the asymptotic admissible set and the limiting variational problem (the Г-limit) have been obtained: [6], [7], [2], [19], [13], [18], [17], [10].

Problem 2 was introduced by Rivière and Serfaty [26] in the context of thin ferromagnetic films. Here $m$ is the magnetization; see for instance [12] for thinfilm models in ferromagnetism. The results for Problem 2 are stronger than for Problem 1 (see [26], [25], [21], [5] and [4]). This might be related to the fact that there are no vortices on the $\varepsilon$-level, which leads to a tighter control of the asymptotic admissibility set. Since vortices play an important role in micromagnetics, Alouges, Rivière and Serfaty [1] have introduced a slight variation of (1) (where 
the penalization of $|m|^{2}-1$ is stronger than the one of $\nabla \cdot m$ ) which allows for vortices on the $\varepsilon$-level - and therefore has more the character of Problem 1.

One might wonder whether we give up too much information by replacing the asymptotic admissible set of Problem 1 or 2 by $A(\Omega)$. Indeed, it can be seen from making $C_{\Phi}$ in (7) more explicit that the measures $\mu_{\Phi}$ enjoy a weak form of uniform control in $\Phi$. The kinetic formulations (see [18] and [25]) quantify this uniform control. But this uniform control differs from problem to problem and would not substantially simplify our proof. This is why we stick to the more flexible $A(\Omega)$.

Parallel to but independently from us, Ambrosio, Kirchheim, Lecumberry and Rivière [4] have proved the same result for a set $\tilde{A}(\Omega)$ which contains the asymptotic admissible set of Problem 2. $\tilde{A}(\Omega)$ is potentially different from $A(\Omega)$ : next to $(2)$, its definition is based on a phase $\theta$, see (4). Their class of entropies $\Phi$ are functions of the phase $\theta$, and not just of $m$ - which is appropriate for Problem 2. As a particular consequence, $\nabla \cdot[\Phi(m)] \neq 0$ for their entropies if $m$ is a vortex $m(x)=\left(-x_{2}, x_{1}\right) /|x|$, whereas our entropies are oblivious to a vortex - as they should be for Problem 1. In this sense, our entropies yield less control than their entropies. This is reflected in the fact that our class of possible blow-ups is a priori richer than theirs, so that we need more arguments to rule most of them out. The proof of [4] is shorter and uses different methods, in particular based on a comparison among certain maps in $\tilde{A}(\Omega)$ and viscosity solutions of the eikonal equation (see [5]).

\subsection{Outlook}

One might wonder what the difficulties in this problem are. In our opinion the difficulties come from the fact that the asymptotic admissible sets for Problem 1 and 2, and a fortiori $A(\Omega)$, are not subsets of vector fields of bounded variation: in [2], an example of an asymptotically admissible $m$ for Problem 1 which is not in BV is given. To be more precise, the paper [2] only establishes that $\nabla \cdot[\Phi(m)]$ is a Radon measure for the Jin-Kohn entropies $\Phi$, but the approximation argument introduced in [9] can be used to show that $m$ is indeed in the asymptotic admissible set. In [26] some evidence was given that a similar example can be constructed for Problem 2.

The reason for this phenomenon lies in the fact that the total variation of the measures $\mu_{\Phi}$ only control the cube $\left|m^{+}-m^{-}\right|^{3}$ of the jump size $\left|m^{+}-m^{-}\right|-$ for BV, one would have to control $\left|m^{+}-m^{-}\right|$itself. This is reflected by (6). The cubic control, which is bad for small jumps, should not be dissociated from (2) - only taken together they give a certain rigidity. Hence our problem is far from a Modica-Mortola scenario.

One might wonder whether this is a problem of broader interest. In a joint work with Michael Westdickenberg [11] suitable modifications of our methods allow us to establish an analogous result for entropy solutions of genuinely nonlinear multidimensional scalar conservation laws. We think that the same could be true even for system with simple structure. What would be the merit of such a result? After all, at 
least for scalar conservation laws, the solution is of bounded variation if the initial data are. The merit rather would consist in pinning down the regularizing effect of nonlinearity. The traditional method which achieves this for multi-dimensional scalar conservation laws is based on the kinetic formulation [22] and velocity averaging [15]. Unfortunately, the linear function space which encodes this gain in regularity is far from BV. Our method could be an alternative route to uncover this regularizing mechanism of nonlinearity in terms of structure properties of the solution. Again, the problem with the linear approach is that the entropy production measure (the analogue of $\mu_{\Phi}$ ), only controls the cube of the jump size (the analogue of $\left.\left|m^{+}-m^{-}\right|\right)$, as is generic for conservation laws. Our approach is oblivious to this inherent nonlinearity.

Acknowledgements. We would like to thank Luigi Ambrosio, Pierre-Emmanuel Jabin, Bernd Kirchheim and Benoît Perthame for many helpful conversations. In particular, discussions with Kirchheim on related measure-theoretic questions (see Sect. 9) encouraged us to come up with the (non-measure-theoretic) arguments of Sect. 7. Felix Otto acknowlegdes partial support by the German Science Foundation through the SFB 611 "Singular phenomena and scaling in mathematical models" at the University of Bonn.

\section{Overview of the proof}

Since the proof of Theorem 1 is lengthy and consists of several parts, we give an outline. We first introduce some language for blow-ups of $m$.

Definition 3. We call

(a) vortex any vector field which up to translation is equal to $m(x)={ }^{\perp} x /|x|$ or $m(x)=-{ }^{\perp} x /|x|$;

(b) line-roof any vector field $m \in A\left(\mathbf{R}^{2}\right)$ which, up to rotation and translation, is equal to

$$
m(x)=\left\{\begin{array}{lll}
m^{+} & \text {if } & x_{1}>0 \\
m^{-} & \text {if } & x_{1}<0
\end{array}\right.
$$

for some choice of constants $m^{+}$and $m^{-}$;

(c) half-roof any $m \in A\left(\mathbf{R}^{2}\right)$ which coincides with a vortex inside a sector and with a line-roof outside, see Fig. 1;

(d) segment-roof any $m \in A\left(\mathbf{R}^{2}\right)$ which coincides with a vortex in a sector $A$, with another vortex in a sector $B$ and with a line-roof in the remaining portion of the plane, see Fig. 1.

A generic field in (b), (c) and (d) will be called a roof.

The sets of fields introduced in (c) and (d) are nonempty, as can be seen from Fig. 1. In this figure, the thick segment represents the jump set of $m$ and the thin rays represent the characteristics of $m$, that is, the rays along which $m$ is constant and normal. Here we use the language of first order equations, see (2) resp. (4). 

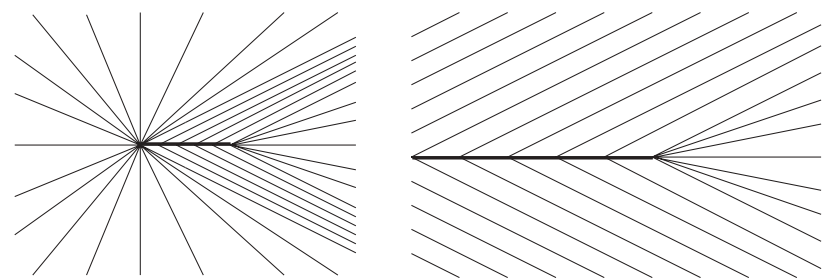

Fig. 1. The singular sets and the characteristics of a segment-roof (on the left) and of a half-roof (on the right)

\section{Definition 4.}

(a) For any $x \in \Omega, r>0$, a field $m$ and measure $\mu$ we introduce the rescalings

$$
m_{x, r}(y)=m(x+r y) \text { and } \mu^{x, r}(A)=\mu(r A+x) .
$$

If $\mu_{\Phi}$ is the entropy measure of $m \in A(\Omega)$ with respect to $\Phi, \frac{1}{r} \mu_{\Phi}^{x, r}$ is the corresponding entropy measure for $m_{x, r}$.

(b) A field $m^{\infty}$ will be called a blow-up of $m$ in $x$ if there exists a sequence $r_{n} \downarrow 0$ such that $\left\{m_{x, r_{n}}\right\}_{n \uparrow \infty}$ converges to $m^{\infty}$ in $L_{\text {loc }}^{p}\left(\mathbf{R}^{2}\right)$ for all $p<\infty$.

(c) $B^{\infty}(x)$ denotes the set of all blow-ups of $m$ in $x$.

Our proof is a combination of general measure theoretic arguments, arguments from Geometric Measure Theory and specific geometric reasoning. We start by a measure theoretic argument in Sect. 3. We interpret the family $\left\{\mu_{\Phi}\right\}_{\Phi}$ of entropy measures as a single measure on $\Omega$ with values in the space of linear forms $T$ on the space of entropies $\Phi$. This allows us to use an infinite-dimensional polar factorization of $\left\{\mu_{\Phi}\right\}_{\Phi}$ into an $x$-dependent family of linear forms $\left\{T_{x}\right\}_{x \in \Omega}$ on $\Phi$-space and a nonnegative measure $v$ on $\Omega$. Roughly speaking, up to an $\mathcal{H}^{1}$-negligible set, we split $\Omega$ into two sets $G \backslash J$ and $J$ which are characterized as follows

$-G \backslash J$ consists of $x$ with

$$
\underset{r \downarrow 0}{\limsup } \frac{1}{r}\left\|\mu_{\Phi}^{x, r}\right\|=0 \quad \text { for all entropies. }
$$

$-J$ consists of $x$ with

$$
\begin{gathered}
\underset{r \downarrow 0}{\limsup } \frac{1}{r}\left\|\mu_{\Phi}^{x, r}\right\|\left\{\begin{array}{c}
<\infty \text { for all entropies } \\
>0 \text { for some entropies }
\end{array}\right\} \\
x \text { is Lebesgue point of }\left\{T_{x}\right\}_{x \in \Omega .} .
\end{gathered}
$$

The compactness results [2], [13] imply that the control (12) resp. (13) yields for all $y \in G$

$$
\left\{m_{y, r}\right\}_{r \downarrow 0} \text { is precompact in } L_{l o c}^{p} \text { for every } p<\infty .
$$


Hence for $y \in G \backslash J$, any $m^{\infty} \in B^{\infty}(y) \subset A\left(\mathbf{R}^{2}\right)$ satisfies

$$
\nabla \cdot\left[\Phi\left(m^{\infty}\right)\right]=0 \text { for all entropies. }
$$

According to [17] this yields

$$
B^{\infty}(y) \subset\{\text { constants, vortices }\} \text { for all } y \in G \backslash J .
$$

In view of (14), we expect that for any $y \in J$ and $m^{\infty} \in B^{\infty}(y) \subset A\left(\mathbf{R}^{2}\right)$

$$
\nabla \cdot\left[\Phi\left(m^{\infty}\right)\right]=T_{y}(\Phi) v^{\infty} \text { for all entropies, }
$$

where $v^{\infty}$ is a nonnegative measure on $\mathbf{R}^{2}$. Hence the information we gain after blow-up is that the family of entropy measures factorizes into a $\Phi$-dependent part $T_{y}$ and an $x$-dependent part $\nu$. If $m \in A\left(\mathbf{R}^{2}\right)$, a linear form $T$ on $\Phi$-space and a nonnegative measure $v$ on $\mathbf{R}^{2}$ satisfy (16), we call the triplet $(m, T, v)$ a split-state. Sections 4, 5, 6 are devoted to the classification of non-degenerate split-states, i. e. $(m, T, v)$ with nontrivial $T \nu$. We will establish that non-degenerate split-states are roofs. We proceed in several steps. In Sect. 4 we prove that $v$ is a rectifiable one-dimensional measure. In Sect. 5 we prove by a second blow-up that the tangent to the rectifiable set which supports $v$ is constant (it only depends on $T$ ). In Sect. 6 we prove that this support is a connected piece of a single line and thus obtain that $m$ is a roof.

The above identification of non-degenerate split-states yields in particular an analogue of (15) for the points of $J$

$$
B^{\infty}(y)\left\{\begin{array}{cc}
\subset & \text { constants, vortices, roofs }\} \\
\not \subset & \text { iconstants, vortices }\}
\end{array}\right\} \quad \text { for all } y \in J .
$$

This information does not yield directly the rectifiability of $J$; we give some reasons for this in Sect. 9. We need to further characterize the set $B^{\infty}(y)$. So in Sect. 7 we also take into account that

- $T_{y}$ in (16) does not depend on $m^{\infty} \in B^{\infty}(y)$

$-m^{\infty} \in B^{\infty}(y)$ are blow-ups of a single field in a single point.

From this we infer that (17) can be improved to

$$
B^{\infty}(y)\left\{\begin{array}{c}
\text { either contains a single line-roof } \\
\text { or contains a single half-roof, } \\
\text { both centered at the origin }
\end{array}\right\} \quad \text { for all } y \in J .
$$

By a similar argument, (15) can be improved to

$$
B^{\infty}(y)\left\{\begin{array}{c}
\text { either contains only constants } \\
\text { or contains a single vortex } \\
\text { centered at the origin }
\end{array}\right\} \quad \text { for all } y \in G \backslash J .
$$

The classification (18) in particular yields a lower bound on the one-dimensional density of $J$. In Sect. 8 we evoke Geometric Measure Theory to conclude rectifiability of $J$. Finally the classification (19) ensures that $m$ has vanishing mean oscillation in all but countably many points of $G \backslash J$. 


\section{Splitting of measures}

In this section we introduce two sets $G$ and $J$ (where $J$ will be the set of Theorem 1 ). Loosely speaking the definition of these sets is based on a "polar factorization" of the distribution-valued measure $\left\{\mu_{\Phi}\right\}_{\Phi}$ and on the approximate continuity of its first factor. This polar factorization is achieved by using differentiation of measures.

Proposition 1. Given $m \in A(\Omega)$ there exist Borel sets $J \subset G$ such that

(a) $J$ is $\mathcal{H}^{1} \sigma$-finite and $\mathcal{H}^{1}(\Omega \backslash G)=0$;

(b) for $x \in G \backslash J B^{\infty}(x)$ consists of constants and vortices;

(c) for $x \in J$ every $m^{\infty} \in B^{\infty}(x)$ satisfies

$$
\nabla \cdot\left[\Phi\left(m^{\infty}\right)\right]=T_{x}(\Phi) v^{\infty} \quad \text { for every entropy } \Phi,
$$

where $T_{x}$ is a distribution which only depends on the point $x$, though the measure $v^{\infty}$ could depend on $m^{\infty}$; moreover $T_{x} \neq 0$ and there exists at least one $m^{\infty} \in B^{\infty}(x)$ such that $v^{\infty} \neq 0$;

(d) if $H \subset \Omega \backslash J$ and $\mathcal{H}^{1}(H)<\infty$ then $\mu_{\Phi}(H)=0$ for every entropy $\Phi$.

Warning 1. The definition of $J$ potentially may depend on the selection of a countable dense subset of the space of all entropies endowed with the $C^{0}\left(\mathbf{S}^{1}\right)-$ norm. So here and in the sequel we fix a countable family $\mathcal{C}:=\left\{\Phi_{i}\right\}_{i \in \mathbf{N}}$ with such a density property and we agree that $\|\Phi\|$ denotes the $C^{0}\left(\mathbf{S}^{1}\right)$-norm of $\Phi$.

Since we will use it several times, we introduce the following notation.

Definition 5. We call split-state every $m \in A\left(\mathbf{R}^{2}\right)$ which satisfies

$$
\nabla \cdot[\Phi(m)]=T(\Phi) v \quad \text { for every entropy } \Phi
$$

for some distribution $T$ on the vector space of entropies and some nonnegative measure $v$. A split-state will be called non-degenerate if $\mu_{\Phi} \neq 0$ for at least one entropy $\Phi$. Moreover with a triplet $(m, T, v)$ we denote an $m \in A\left(\mathbf{R}^{2}\right)$, a nonnegative measure $v$ and a distribution $T$ which satisfy (20).

Using this language point (c) of Proposition 1 becomes

(c) for $x \in J$ every $m^{\infty} \in B^{\infty}(x)$ is a split state and at least one of them is non-degenerate; there exists a unique distribution $T$ such that to every $m^{\infty} \in B^{\infty}(x)$ we can associate a triplet $\left(m^{\infty}, T, v^{\infty}\right)$;

Before addressing the proof we first state some basic properties of rescaling of maps in the class $A(\Omega)$ and possible blow-ups.

Lemma 1. Given $m \in A(\Omega)$ the following holds:

(a) for every entropy $\Phi$ we have

$$
\left\|\mu_{\Phi}\right\| \ll \mathcal{H}^{1}
$$


(b) if we have

$$
\limsup _{r \downarrow 0} \frac{\left\|\mu_{\Phi_{i}}\right\|\left(B_{r}(x)\right)}{r}<\infty \quad \text { for every } \Phi_{i} \in \mathcal{C}
$$

then $\left\{m_{x, r}\right\}_{r \downarrow 0}$ is strongly precompact in $L_{l o c}^{p}\left(\mathbf{R}^{2}\right)$ for $p<\infty$;

(c) if (22) holds and $m_{x, r_{n}} \rightarrow m^{\infty}$ then

$$
\nabla \cdot\left[\Phi_{i}\left(m_{x, r_{n}}\right)\right]=\frac{\mu_{\Phi_{i}}^{x, r_{n}}}{r_{n}} \stackrel{*}{\rightarrow} \nabla \cdot\left[\Phi_{i}\left(m^{\infty}\right)\right]
$$

in the sense of measures.

Proof. First Step Proof of (a).

Since $\mu_{\Phi}=\nabla \cdot[\Phi(m)]$ is the divergence of an $L^{\infty}$ field it is easy to see that the upper one-density of $\mu_{\Phi}$ is finite everywhere. Indeed, testing the identity $\nabla \cdot[\Phi(m)]=\mu_{\Phi}$ with the mollification of the characteristic function of $B_{r}(x)$ we obtain

$$
\left|\mu_{\Phi}\left(B_{r}(x)\right)\right| \leq 2 \pi r\|\Phi\| .
$$

Now for every $x$ such that

$$
\lim _{r \downarrow 0} \frac{\left|\mu_{\Phi}\left(B_{r}(x)\right)\right|}{\left\|\mu_{\Phi}\right\|\left(B_{r}(x)\right)}=1
$$

we thus obtain

$$
\underset{r \downarrow 0}{\limsup } \frac{\left\|\mu_{\Phi}\right\|\left(B_{r}(x)\right)}{r} \leq 2 \pi\|\Phi\| .
$$

Since (25) holds for $\left\|\mu_{\Phi}\right\|$-a.e. $x$, standard arguments in Geometric Measure Theory (see Theorem 2.56 of [3]) imply (21).

Second Step Proof of (b).

If $m \in A(\Omega)$ then $m_{x, r} \in A\left(\Omega_{x, r}\right)$, where $\Omega_{x, r}$ denotes a suitable rescaling of $\Omega$ (and for $r \downarrow 0, \Omega_{x, r} \uparrow \mathbf{R}^{2}$ ). Indeed it is easy to see that

$$
\nabla \cdot\left[\Phi\left(m_{x, r}\right)\right]=\frac{\mu_{\Phi}^{x, r}}{r} .
$$

So slightly modifying the proof of compactness of [13] we conclude that (22) yields $L_{l o c}^{p}$-strong precompactness of $m_{x, r}$ for every $p<\infty$.

Third Step Proof of (c).

If $m_{x, r} \rightarrow m^{\infty}$ strongly in $L_{l o c}^{p}$, then clearly (23) holds in the sense of distributions. Finally (27), (22) and compactness of the weak* topology of the space of Radon measures give that (23) holds in the sense of measures.

Remark 1 . Choosing suitably $\mathcal{C}$ we could have proved (b) using the compactness result of [2]. Indeed there it is proved that a control on two particular entropies is sufficient for compactness and hence for our purposes it would be enough to include these two entropies in $\mathcal{C}$. 
Proof of Proposition 1. First Step After fixing $\mathcal{C}=\left\{\Phi_{i}\right\}$ as in Warning 1 let

$$
X_{N}:=\text { linear span of }\left\{\Phi_{1}, \ldots, \Phi_{N}\right\} \text {; }
$$

we define the vector-valued measure $\mu_{N}$ taking values into $X_{N}^{*}$ (the dual of $X_{N}$ ) as

$$
\left\langle\mu_{N}, \Phi\right\rangle:=\nabla \cdot[\Phi(m)]
$$

To fix ideas we endow $X_{N}$ with the $C^{0}\left(\mathbf{S}^{1}\right)$-norm and $X_{N}^{*}$ with the dual one and we introduce the notation

$$
\left\|\mu_{N}\right\|:=\text { total variation measure of } \mu_{N} .
$$

By the Radon-Nykodim Theorem there exists $U_{N} \in L^{1}\left(\Omega, X_{N}^{*},\left\|\mu_{N}\right\|\right)$ such that $\left\|U_{N}\right\|=1\left\|\mu_{N}\right\|$-a.e. and $\mu_{N}=U_{N}\left\|\mu_{N}\right\|$.

Second Step We introduce the following two families of sets:

$$
\begin{aligned}
& S_{N}:=\left\{x \in \Omega \mid \limsup _{r \downarrow 0} \frac{\left\|\mu_{N}\right\|\left(B_{r}(x)\right)}{r}>0\right\}, \\
& L_{N}:=\left\{x \in \Omega \mid x \text { is a Lebesgue point for } U_{N}\right\} .
\end{aligned}
$$

We start by collecting some properties of the $S_{N}$ 's. From the ordering of $X_{N}$ we obtain that

$$
\left\|\mu_{N}\right\| \leq\left\|\mu_{N^{\prime}}\right\| \quad \text { for every pair } N<N^{\prime} .
$$

This implies that

$$
\forall N<N^{\prime} \quad S_{N} \subset S_{N^{\prime}}
$$

Since the measure $\mu_{N}$ has finite total variation we have by standard arguments (see for example Theorem 2.56 of [3]) that

$$
\forall N \quad S_{N} \text { is } \mathcal{H}^{1} \sigma \text {-finite }
$$

and

$$
\forall N \quad \mathcal{H}^{1}\left\llcornerS _ { N } \ll \| \mu _ { N } \| \left\llcorner S_{N} .\right.\right.
$$

We now turn to the $L_{N}$ 's. Because $L_{N}$ consists of Lebesgue points of $U_{N}$ we have by standard arguments that

$$
\left\|U_{N}(x)\right\|=1 \quad \forall x \in L_{N} .
$$

From the ordering of the $X_{N}$ 's for any pair $N<N^{\prime}$ we obtain that

$$
\left.U_{N^{\prime}}\right|_{X_{N}} \neq 0 \text { and }\left.U_{N^{\prime}}\right|_{X_{N}}=\left\|\left.U_{N^{\prime}}\right|_{X_{N}}\right\| U_{N} \quad \mu_{N^{\prime}} \text { a.e. on }\left\{U_{N} \neq 0\right\} \text {. }
$$


In view of (33) this yields

$$
\left\{\begin{array}{l}
\left.U_{N^{\prime}}(x)\right|_{X_{N}} \neq 0 \\
\left.U_{N^{\prime}}(x)\right|_{X_{N}}=\left\|\left.U_{N^{\prime}}(x)\right|_{X_{N}}\right\| U_{N}(x)
\end{array}\right\} \quad \forall x \in L_{N} \cap L_{N^{\prime}} .
$$

By elementary measure theory we get

$$
\left\|\mu_{N}\right\|\left(\Omega \backslash L_{N}\right)=0 .
$$

Third Step We now define $G$ and $J$ in Proposition 1

$$
\begin{aligned}
G_{0} & :=\Omega \backslash \bigcup_{N \in \mathbf{N}} S_{N} \\
J & :=\bigcup_{N \in \mathbf{N}}\left(S_{N} \cap \bigcap_{N^{\prime} \geq N} L_{N^{\prime}}\right) \\
G & :=J \cup G_{0} .
\end{aligned}
$$

The $\mathcal{H}^{1} \sigma$-finiteness of $J$ follows immediately from (32) and $J \subset \cup S_{N}$. Let us now argue that $\mathcal{H}^{1}(\Omega \backslash G)=0$. We have

$$
\begin{aligned}
& \Omega \backslash G \subset \bigcup_{N \in \mathbf{N}}\left(S_{N} \backslash \bigcap_{N^{\prime} \geq N} L_{N^{\prime}}\right)=\bigcup_{N^{\prime} \in \mathbf{N}}\left(\bigcup_{N \leq N^{\prime}} S_{N} \backslash L_{N^{\prime}}\right) \\
& \stackrel{(30)}{=} \bigcup_{N^{\prime} \in \mathbf{N}}\left(S_{N^{\prime}} \backslash L_{N^{\prime}}\right) .
\end{aligned}
$$

We now observe that for fixed $N^{\prime} \in \mathbf{N}$ we have, according to (35),

$$
\left\|\mu_{N^{\prime}}\right\|\left(S_{N^{\prime}} \backslash L_{N^{\prime}}\right) \leq\left\|\mu_{N^{\prime}}\right\|\left(\Omega \backslash L_{N^{\prime}}\right)=0
$$

and according to (32)

$$
\mathcal{H}^{1} \ll\left\|\mu_{N^{\prime}}\right\| \text { on } S_{N^{\prime}} \supset S_{N^{\prime}} \backslash L_{N^{\prime}}
$$

so that $\mathcal{H}^{1}\left(S_{N^{\prime}} \backslash L_{N^{\prime}}\right)=0$. This proves that $\mathcal{H}^{1}(\Omega \backslash G)=0$ and hence completes the proof of point (a).

Fourth Step We now construct $T$ on $J$. In this step $T(x)$ for $x \in J$ will be constructed as a possible unbounded linear form on $\bigcup_{N} X_{N}$. We will extend it to a bounded linear functional on the space of all entropies in a later step.

Fix $x \in J$ and let $N_{x} \in \mathbf{N}$ be the smallest $N \in \mathbf{N}$ with $x \in S_{N} \cap \bigcap_{N^{\prime} \geq N} L_{N^{\prime}}$. We will renormalize the linear forms $U_{N}(x)$ for $N \geq N_{x}$ so to have that they are extensions of one another. Since $x \in L_{N_{x}}$ and in view of (33) there exists a $\Phi_{x} \in X_{N_{x}}$ such that $\left\|\Phi_{x}\right\|=1$ and $\left\langle U_{N_{x}}(x), \Phi_{x}\right\rangle=1$.

Since for $N \geq N_{x}$ we have $x \in L_{N} \cap L_{N_{x}}$, from (34) we conclude

$$
\left\langle U_{N}(x), \Phi_{x}\right\rangle \neq 0 \quad \text { for any } N \geq N_{x} .
$$


We use the value in $\Phi_{x}$ to renormalize the linear forms $U_{N}(x)$ :

$$
T_{N}(x):=\frac{1}{\left\langle U_{N}(x), \Phi_{x}\right\rangle} U_{N}(x)
$$

so that we have

$$
\left\langle T_{N}(x), \Phi_{x}\right\rangle=1 \text {. }
$$

Since $x \in L_{N} \cap L_{N^{\prime}}$ for $N^{\prime} \geq N \geq N_{x}$, (38) and (34) imply that

$$
\left.T_{N^{\prime}}(x)\right|_{X_{N}}=T_{N}(x) \text {. }
$$

Hence there exists a linear form $T(x)$ on the union of all $X_{N}$ 's such that

$$
\left.T(x)\right|_{X_{N}}=T_{N}(x) \quad \text { for all } N \geq N_{x} .
$$

Fifth Step We now study the blow-ups in a point $x \in G_{0}$. We have by definition of $G_{0}$ that

$$
\lim _{r \downarrow 0} \frac{\left\|\mu_{N}\right\|\left(B_{r}(x)\right)}{r}=0 \quad \text { for all } N \in \mathbf{N} \text {. }
$$

Hence (b) in Lemma 1 implies that $\left\{m_{x, r}\right\}_{r \downarrow 0}$ is strongly precompact in $L_{l o c}^{p}$ for $p<\infty$. Moreover for all $i$ (40) holds with $\left\|\mu_{\Phi_{i}}\right\|$ in place of $\left\|\mu_{N}\right\|$, which translates into

$$
\frac{\mu_{\Phi_{i}}^{x, r}}{r} \stackrel{*}{\rightarrow} 0 .
$$

Hence we obtain for every blow-up $m^{\infty}$ that $\nabla \cdot\left[\Phi_{i}\left(m^{\infty}\right)\right]=0$. Since $\mathcal{C}$ is $C^{0}\left(\mathbf{S}^{1}\right)-$ dense in the set of entropies we have $\nabla \cdot\left[\Phi\left(m^{\infty}\right)\right]=0$ for all entropies $\Phi$. Now the results in [17] imply that every $m^{\infty} \in B^{\infty}(x)$ is either a constant or a vortex and hence gives point (b).

Sixth Step We now study the blow-ups in a point $x \in J$. We notice that (25) holds since $x$ is a Lebesgue point for $U_{N}$. Hence (b) in Lemma 1 implies that $\left\{m_{x, r}\right\}_{r \downarrow 0}$ is precompact in the strong $L_{l o c}^{p}$ topology.

Let us fix $m^{\infty} \in B^{\infty}(x)$ and a sequence of radii $r_{n} \downarrow 0$ such that $m_{x, r_{n}} \rightarrow m^{\infty}$ in $L_{l o c}^{p}$. Moreover recall the definitions of $N_{x}$ and $\Phi_{x}$ given in the Fourth Step.

Without loosing our generality we may assume that for some $N \in \mathbf{N}$

$$
\limsup _{n \rightarrow \infty} \frac{\left\|\mu_{N}\right\|\left(B_{r_{n}}(x)\right)}{r_{n}}>0
$$

otherwise reasoning as in the previous step we would have $\nabla \cdot\left[\Phi\left(m^{\infty}\right)\right]=0$ for every entropy $\Phi$ and hence $m^{\infty}$ would be a degenerate split-state.

Let $N_{*}$ be the smallest integer satisfying (41). According to our definition $N_{*} \geq N_{x}$. After passing to a subsequence we can assume that

$$
\liminf _{n \rightarrow \infty} \frac{\left\|\mu_{N_{*}}\right\|\left(B_{r_{n}}(x)\right)}{r_{n}}>0
$$


which, thanks to (29), implies

$$
\liminf _{n \rightarrow \infty} \frac{\left\|\mu_{N}\right\|\left(B_{r_{n}}(x)\right)}{r_{n}}>0 \quad \forall N \geq N_{*} .
$$

Let us now fix $N \geq N_{*}$. Thanks to (43) we can assume, passing to another subsequence, that there exists a nonnegative measure $v_{N} \neq 0$ such that

$$
\left(\frac{\left\langle U_{N}(x), \Phi_{x}\right\rangle\left\|\mu_{N}\right\|^{x, r_{n}}}{r_{n}}\right) \stackrel{*}{\rightarrow} v_{N} .
$$

According to the definition of $J$ and because of $N \geq N_{*} \geq N_{x}, x$ is a Lebesgue point for $U_{N}$. Hence we have

$$
\lim _{r \downarrow 0} \frac{1}{\left\|\mu_{N}\right\|\left(B_{r}(x)\right)} \int_{B_{r}(x)}\left\|U_{N}(y)-U_{N}(x)\right\| d\left\|\mu_{N}\right\|(y)=0
$$

which thanks to (43) implies

$$
\lim _{r \downarrow} \frac{1}{r_{n}} \int_{B_{r_{n}}(x)}\left\|U_{N}(y)-U_{N}(x)\right\| d\left\|\mu_{N}\right\|(y)=0 .
$$

This last equation yields that

$$
\frac{\mu_{N}^{x, r_{n}}}{r_{n}}-\frac{U_{N}(x)\left\|\mu_{N}\right\|^{x, r_{n}}}{r_{n}} \stackrel{*}{\rightarrow} 0
$$

in the sense of measures. Now for every $\Phi \in X_{N}$ we may write

$$
\nabla \cdot\left[\Phi\left(m_{x, r_{n}}\right)\right]=\left\langle U_{N}, \Phi\right\rangle \frac{\left\|\mu_{N}\right\|^{x, r_{n}}}{r_{n}}
$$

and hence from (45) together with (44) we obtain

$$
\nabla \cdot\left[\Phi\left(m_{x, r_{n}}\right)\right] \stackrel{*}{\rightarrow} \frac{\left\langle U_{N}(x), \Phi\right\rangle}{\left\langle U_{N}(x), \Phi_{x}\right\rangle} v_{N} \stackrel{(37,39)}{=}\langle T(x), \Phi\rangle v_{N} .
$$

Since from $(39,38)\left\langle T(x), \Phi_{x}\right\rangle=1$, we see that $v_{N}$ does not depend on $N$ and so we define $v:=v_{N}$. Hence we have

$$
\nabla \cdot\left[\Phi\left(m^{\infty}\right)\right]=\langle T(x), \Phi\rangle v \quad \forall \Phi \in \bigcup_{N \in \mathbf{N}} X_{N} .
$$

Since $v \neq 0$ there exists $\zeta \in C_{c}^{\infty}\left(\mathbf{R}^{2}\right)$ with $\int \zeta d v=1$ and so we have

$$
\langle T(x), \Phi\rangle=-\int \Phi\left(m^{\infty}(y)\right) \cdot \nabla \zeta(y) d y .
$$

We conclude that $T(x)$ is bounded with respect to the $C^{0}\left(\mathbf{S}^{1}\right)-$ norm. Since with respect to this norm $\bigcup X_{N}$ is dense in the set of all entropies we can extend $T$ in a unique way to a bounded linear functional on the space of entropies endowed with the $C^{0}\left(\mathbf{S}^{1}\right)$-norm. This implies that (46) holds for every entropy $\Phi$ and hence concludes the proof of point (c). 
Seventh Step We now come to the proof of (d). Let us fix $H \subset \Omega \backslash J$ such that $\mathcal{H}^{1}(H)<\infty$ and suppose that $\Phi$ is a given entropy. From the previous steps we know that for $\mathcal{H}^{1}$-a.e. $x \in H \quad B^{\infty}(x)$ is made of degenerate split states. So we conclude that for every sequence $r_{n} \downarrow 0$ we have $\nabla \cdot\left[\Phi\left(m_{x, r_{n}}\right)\right] \stackrel{*}{\rightarrow} 0$ in the sense of distributions. Hence $\left\{\mu_{\Phi}^{x, r} / r\right\}_{r \downarrow 0}$ converges to 0 in the sense of distributions. But we also recall (see (26)) that

$$
\limsup _{r \downarrow 0} \frac{\left\|\mu_{\Phi}^{x, r}\right\|\left(B_{1}\right)}{r} \leq 2 \pi\|\Phi\| \quad \text { for }\left\|\mu_{\Phi}\right\|-\text { a.e. } x \in H .
$$

This means that $\left\{\mu_{\Phi}^{x, r} / r\right\}_{r \downarrow 0}$ converges to zero in the sense of measures in $\left\|\mu_{\Phi}\right\|-$ a.e. $x \in H$. Reasoning as in the Sixth Step, we can conclude that also $\left\{\left\|\mu_{\Phi}^{x, r}\right\| / r\right\}_{r \downarrow 0}$ converges to 0 in the sense of measures in $\left\|\mu_{\Phi}\right\|-$ a.e. $x \in H$. But since these last measures are nonnegative we infer

$$
\lim _{r \downarrow 0} \frac{\left\|\mu_{\Phi}\right\|\left(B_{r}(x)\right)}{r}=0 \quad \text { for }\left\|\mu_{\Phi}\right\|-\text { a.e. } x \in H
$$

which (see for example Theorem 2.56 of [3]) implies $\left\|\mu_{\Phi}\right\|(H)=0$.

\section{Rectifiability for split measures}

In this section we start with the classification of the non-degenerate split states $(m, T, v)$.

Proposition 2. Let $(m, T, v)$ be a non-degenerate split-state. Then $v$ is supported on a closed rectifiable one-dimensional set, therefore it is a rectifiable onedimensional measure.

We achieve this by using a certain family of "generalized entropies" which are discontinuous but pointwise limits of smooth entropies. These generalized entropies were first introduced in [13] to study the compactness for the variational problem (10) and they are very similar to the ones introduced by Kruzkov in the theory of scalar conservation laws, [20].

Proposition 3. Using polar coordinates for $(\xi, z) \in \mathbf{S}^{1} \times \mathbf{S}^{1}$ (i.e. $\xi=e^{i \alpha}$ and $\left.z=e^{i \theta}\right)$ we define the "Kruzkov functions" $\chi(\xi, z)$ as

$$
\chi(\xi, z)=\left\{\begin{array}{l}
1 \text { if }(\theta-\alpha) \in[2 k \pi,(2 k+1) \pi[\text { for some } k \in \mathbf{Z} \\
0 \text { otherwise. }
\end{array}\right.
$$

If $(m, T, v)$ is a split state, then for every $\xi \in \mathbf{S}^{1}$ we have

$$
\xi \cdot \nabla_{x}[\chi(\xi, m)]=f(\xi) v \text { distributionally on } \mathbf{R}^{2} .
$$

Moreover $f(-\xi)=f(\xi)$ and $f \in B V\left(\mathbf{S}^{1}\right)$. 
Equation (47) is a particular example of a kinetic formulation of a scalar conservation law, see [22]. As in the traditional setting of scalar conservation laws, the kinetic formulation encodes the information of all Kruzkov entropies. Its merit is that it encapsulates the characteristics for the first order equation (2): a smooth $m$ would be constant along the lines perpendicular to $m$. The right hand side of (47) measures the deviation from this geometric principle. The kinetic formulation for Problem 1 has first been introduced by Jabin \& Perthame in [18], see also [25]. (47) slightly differs from [18] since we start from a split-state instead of a general $m \in A(\Omega)$ : our right hand side is more regular and hence can be written without distributional derivatives in $\xi$.

Proposition 3 will be used to prove the following

Lemma 2. There exists $\alpha>0$ such that if $x$ is a Lebesgue point of $m$ then there is a one-sided open cone $C_{x}$ of opening $\alpha$ and vertex $x$ such that $v\left(C_{x}\right)=0$.

We see below that this Lemma easily implies the rectifiability of $v$.

Proof of Proposition 2. Let us fix $x \in \mathbf{R}^{2}$ and take a sequence of points $\left\{x_{n}\right\}_{n \uparrow \infty}$ which are Lebesgue for $m$ and converge to $x$. Possibly passing to a subsequence the cones $C_{x_{n}}$ of Lemma 2 converge to an open cone $C_{x}$ of opening $\alpha$ with vertex in $x$. Hence $v\left(C_{x}\right)=0$. Take now the closed set $S=\operatorname{supp}(v)$. We can find a finite family of closed sets $S_{i}$ and unit vectors $\xi_{i}$ such that

(i) $S_{i} \subset S$ and $\bigcup S_{i}=S$;

(ii) $\forall x \in S_{i}$, if $C_{x}^{\prime}:=\left\{x+y: \alpha|y| / 2 \leq y \cdot \xi_{i}\right\}$ then $v\left(C_{x}^{\prime}\right)=0$.

This gives that $C_{x}^{\prime} \cap S_{i}=\emptyset$ for every $x \in S_{i}$ (because $v\left(C_{x}^{\prime}\right)=0$ and $\left.S_{i} \subset \operatorname{supp}(v)\right)$. Hence $S_{i}$ is contained in the graph of a Lipschitz function.

The remaining part of the section is devoted to proving Lemma 2 and Proposition 3 above.

Remark 2. In the following we fix an orientation for $\mathbf{S}^{1}$, e.g. the counterclockwise one. Moreover if $\xi, \xi_{1}, \xi_{2} \in \mathbf{S}^{1}$ and the angle between $\xi$ and $\xi_{1}$ is positive and strictly less than the angle between $\xi_{2}$ and $\xi_{1}$ then we write $\left.\xi \in\right] \xi_{1}, \xi_{2}[$.

Proof of Proposition 3. First Step Let $\xi \in \mathbf{S}^{1}$ be given. Reasoning as in Lemma 4 of [13] one can prove that there exists a sequence of entropies $\Psi_{n}$ such that $\left\|\Psi_{n}\right\|$ is equibounded and $\Psi_{n}(x) \rightarrow \xi \chi(\xi, x)$ for every $x$. Now thanks to the fact that $T$ of equation (20) is a bounded linear functional on $C^{0}\left(\mathbf{S}^{1}\right)$ we can pass to the limit in $\nabla \cdot\left[\Psi_{n}(m)\right]=T\left(\Psi_{n}\right) v$ to get $\nabla \cdot[\xi \chi(\xi, m)]=f(\xi) v$ for some real number $f(\xi)$. A trivial computation gives the homogeneity of $f$.

Second Step We now come to the proof that $f$ is a BV function. First of all we take a function $\varphi \in C_{c}^{1}\left(\mathbf{R}^{2}\right)$ such that $\int \varphi d v=1$. We will prove that if the angle between $\xi_{1}, \xi_{2} \in \mathbf{S}^{1}$ is less than $\pi$, then $f$ is of bounded variation in $] \xi_{1}, \xi_{2}[$. 
We fix $\xi_{1}$ and $\xi_{2}$ and for every $\xi$ we call $\theta(\xi)$ the angle between $\xi_{1}$ and $\xi$. Pick up $z_{1}, \ldots, z_{n}$ such that $\xi_{1} \leq z_{1}<z_{2}<\ldots<z_{n} \leq \xi_{2}$. Using equation (47) we get

$$
\begin{aligned}
\left|f\left(z_{i}\right)-f\left(z_{i-1}\right)\right|= & \left|\int_{\mathbf{R}^{2}} z_{i} \cdot \nabla \varphi \chi\left(z_{i}, m\right)-\int_{\mathbf{R}^{2}} z_{i-1} \cdot \nabla \varphi \chi\left(z_{i-1}, m\right)\right| \\
\leq & \left|\int_{\mathbf{R}^{2}}\left(z_{i}-z_{i-1}\right) \cdot \nabla \varphi \chi\left(z_{i}, m\right)\right| \\
& +\left|\int_{\mathbf{R}^{2}} z_{i-1} \cdot \nabla \varphi\left(\chi\left(z_{i}, m\right)-\chi\left(z_{i-1}, m\right)\right)\right| \\
\leq & \left|z_{i}-z_{i-1}\right|\|\nabla \varphi\|_{L^{1}}+\|\nabla \varphi\|_{L^{\infty}} \mathcal{L}^{2}\left(\operatorname{supp}(\varphi) \cap S_{i}\right)
\end{aligned}
$$

where

$$
S_{i}=\left\{\begin{array}{l|l}
x & \begin{array}{l}
\text { either } \theta\left(z_{i}\right) \leq \theta(m(x))<\theta\left(z_{i+1}\right) \\
\text { or } \pi+\theta\left(z_{i}\right) \leq \theta(m(x))<\pi+\theta\left(z_{i+1}\right)
\end{array}
\end{array}\right\} .
$$

Notice that, since the angle between $\xi_{1}$ and $\xi_{2}$ is less than $\pi$, the sets $S_{i}$ are all disjoint. Hence we find that

$$
\sum\left|f\left(z_{i}\right)-f\left(z_{i}-1\right)\right| \leq\left|\xi_{2}-\xi_{1}\right|\|\nabla \varphi\|_{L^{1}}+\|\nabla \varphi\|_{L^{\infty}} \mathcal{L}^{2}(\operatorname{supp}(\varphi))
$$

and this completes the proof.

We are now ready to prove Lemma 2 and end this section. Before doing it we will give the heuristic explanation which is hidden in the proof. Let $x$ be a given Lebesgue point for $m$. Thanks to what proved so far there is a sector $G \subset \mathbf{S}^{1}$ such that

$$
\chi(\xi, m(x))=1 \quad \text { and } \quad \xi \cdot \nabla_{x}(\chi(\xi, m))=f(\xi) \nu \geq 0 \quad \forall \xi \in G .
$$

So if we call $r_{\xi}$ the half-line starting from $x$ and directed along $\xi$ we have $\chi\left(\xi, m\left(x^{\prime}\right)\right)=1$ for every $x^{\prime} \in r_{\xi}$. Loosely speaking this tells us that $r_{\xi}$ "does not meet" the measure $v$. Since this happens for every $\xi \in G$ one would like to conclude that $\nu$ is identically zero inside the cone given by $\bigcup_{\xi \in G} r \xi$.

Proof of Lemma 2. From the condition $\nabla \cdot m=0$, integrating on $\mathbf{S}^{1}$ both sides of equation (47) we get $\int_{\mathbf{S}^{1}} f(\xi)=0$. Since $m$ corresponds to a non-degenerate split-state it cannot be $f=0$. Hence there must be a measurable subset on which $f$ is positive. Thanks to Proposition $3 f$ is continuous except for an at most countable number of points. So we can choose a $\xi$ for which $f$ is positive in an interval containing $\xi$. Thanks to its homogeneity, $f$ is positive even on an interval containing $-\xi$. As a consequence we have that there exist $\alpha, \beta, \gamma>0$ such that for every $w \in \mathbf{S}^{1}$ there is a couple $\xi_{1}, \xi_{2}$ which satisfies:

(i) $f(\xi) \geq \beta$ for every $\xi \in] \xi_{1}, \xi_{2}[$;

(ii) the cone individuated by $\xi_{1}$ and $\xi_{2}$ has opening bigger than $\alpha$;

(iii) $w \cdot \xi \geq \gamma>0$ for every $\xi \in] \xi_{1}, \xi_{2}[$. 
Hence we now take as $w$ the unique element of $\mathbf{S}^{1}$ such that

$$
\lim _{r \rightarrow 0} \frac{1}{r^{2}} \int_{B_{r}(x)}|m(y)-w| d y=0
$$

(which exists thanks to the fact that $x$ is a Lebesgue point for $m$ ) and we chose $\xi_{1}$ and $\xi_{2}$ which satisfy the three conditions above. Moreover for the sake of simplicity we will denote $w$ by $m(x)$.

We claim that if we consider the cone

$$
C_{x}:=\{x+r \xi \mid \xi \in] \xi_{1}, \xi_{2}[, r>0\},
$$

then $v\left(C_{x}\right)=0$. We will prove this in several steps.

First Step We fix $\rho>0$ and for every $\varepsilon>0$ and every $\xi \in] \xi_{1}, \xi_{2}\left[\right.$ we call $R_{\varepsilon}^{\xi}$ (see Fig. 2 below) the open set made by the union of:

(i) the rectangle given by $\left\{x+a \xi+b^{\perp} \xi\right\}$, with $b \in(-\varepsilon, \varepsilon), a \in(0, \rho)$;

(ii) the ball $B_{\varepsilon}(x)$ and the ball $B_{\varepsilon}(x+\rho \xi)$.

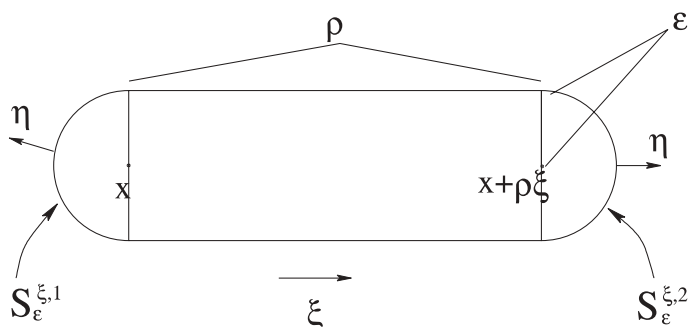

Fig. 2. The open set $R_{\varepsilon}^{\xi}$

The boundary of $R_{\varepsilon}^{\xi}$ is made of two segments parallel to $\xi$ and two half-circles. We call $S_{\varepsilon}^{\xi, 1}$ the half-circle centered at $x, S_{\varepsilon}^{\xi, 2}$ the half-circle centered at $x+\rho \xi$ and $\eta$ the exterior unit normal to $\partial R_{\varepsilon}^{\xi}$. We now want to estimate $v\left(R_{\varepsilon}^{\xi}\right)$. We take a standard family of mollifiers $\psi_{\delta}$ and we recall that, since $v$ is nonnegative,

$$
v\left(R_{\varepsilon}^{\xi}\right) \leq \lim _{\delta \rightarrow 0} v * \psi_{\delta}\left(R_{\varepsilon}^{\xi}\right)
$$

(indeed we have $v * \psi_{\delta}\left(R_{\varepsilon}^{\xi}\right)=\int \psi_{\delta} * \chi_{R_{\varepsilon}^{\xi}} d v$ and, since $\psi_{\delta} * \chi_{R_{\varepsilon}^{\xi}}(x) \uparrow 1$ on every $x \in R_{\varepsilon}^{\xi}$, letting $\delta \downarrow 0$ we get (48)). Since $f(\xi) \geq \beta$ we obtain

$$
\begin{aligned}
\beta v * \psi_{\delta}\left(R_{\varepsilon}^{\xi}\right) & \leq f(\xi) v * \psi_{\delta}\left(R_{\varepsilon}^{\xi}\right)=\int_{R_{\varepsilon}^{\xi}} \xi \cdot \nabla_{x}\left(\chi(\xi, m) * \psi_{\delta}\right) \\
& =\int_{S_{\varepsilon}^{\xi, 2} \cup S_{\varepsilon}^{\xi, 1}}\left(\chi(\xi, m) * \psi_{\delta}\right) \xi \cdot \eta \\
& \leq \int_{S_{\varepsilon}^{\xi, 2}} \xi \cdot \eta+\int_{S_{\varepsilon}^{\xi, 1}}\left(\chi(\xi, m) * \psi_{\delta}\right) \xi \cdot \eta
\end{aligned}
$$

where the last inequality comes from the fact that $\xi \cdot \eta \geq 0$ on $S_{\varepsilon}^{\xi, 2}$ and $\chi(\xi, m) \leq 1$. 
Second Step For every set $B$, call $\operatorname{Leb}(B)$ the set of Lebesgue points for $m$ which belong to $B$. Standard arguments involving Fubini-Tonelli Theorem imply that there is a sequence $\varepsilon_{n} \downarrow 0$ such that

$$
\frac{\mathcal{H}^{1}\left(\operatorname{Leb}\left(\partial B_{\varepsilon_{n}}(x)\right)\right)}{2 \pi \varepsilon_{n}}=1 \text {. }
$$

Moreover, from the fact that $m(x) \cdot \xi \geq \gamma>0$ and using again Fubini-Tonelli Theorem, it is easy to see that we can choose $\varepsilon_{n}$ so that

$$
\frac{\mathcal{H}^{1}\left(\operatorname{Leb}\left(\partial B_{\varepsilon_{n}}(x)\right) \cap\{y \mid \chi(\xi, m(y))=1\}\right)}{2 \pi \varepsilon_{n}} \rightarrow 1
$$

uniformly in $\xi \in] \xi_{1}, \xi_{2}$ [. Going back to (49), if we let $\delta \downarrow 0$ we gain

$$
\beta \nu\left(R_{\varepsilon_{n}}^{\xi}\right) \leq\left[\int_{S_{\varepsilon_{n}}^{\xi, 2}} \xi \cdot \eta+\int_{S_{\varepsilon_{n}}^{\xi, 1}} \xi \cdot \eta\right]+o\left(\varepsilon_{n}\right)=o\left(\varepsilon_{n}\right)
$$

Third Step If we integrate on $\xi$ both sides of (50) we obtain

$$
\beta \int_{\xi^{1}}^{\xi_{2}} \nu\left(R_{\varepsilon_{n}}^{\xi}\right) \leq \alpha o\left(\varepsilon_{n}\right)
$$

from which, dividing by $\varepsilon_{n}$ and changing the order of integration, we get

$$
\lim _{n \rightarrow \infty} \beta \int_{\mathbf{R}^{2}}\left[\int_{\xi 1}^{\xi_{2}} \frac{\chi_{R_{\varepsilon_{n}}^{\xi}}(y)}{\varepsilon_{n}} d \xi\right] d \nu(y)=0 .
$$

We notice that the sequence of functions

$$
g_{n}(y):=\int_{\xi^{1}}^{\xi_{2}} \frac{\chi_{R_{\varepsilon_{n}}^{\xi}}(y)}{\varepsilon_{n}} d \xi
$$

converges to $2 \alpha /|y-x|$ in every $y \in C_{x}^{\rho}:=\{x+r \xi: \xi \in] \xi_{1}, \xi_{2}[, 0<r<\rho\}$. We then have

$$
\beta \int_{C_{x}^{\rho}} \frac{2 \alpha}{|y-x|} d \nu(y)=0 .
$$

Hence $v\left(C_{x}^{\rho}\right)=0$, which (letting $\left.\rho \uparrow \infty\right)$ gives $v\left(C_{x}\right)=0$. 


\section{Blow-up for non-degenerate split-states}

In this section we continue to investigate split-states $(m, T, v)$. According to the last section the blow-ups of $v$ are given ( $v-$ a.e.) by multiples of the Hausdorff measure concentrated on lines. In this section we will show that this forces the blow-up of $m$ to be a line-roof in $\mathcal{H}^{1}$-a.e. point where $v$ is concentrated. Furthermore this line-roof only depends on $T$.

Proposition 4. Let $(m, T, v)$ be a non-degenerate split-state. Then there exists a line-roof $m^{T}$, determined by $T$, such that $B^{\infty}(x)=\left\{m^{T}\right\}$ for $v$-a.e. $x$. Hence we may assume (after possibly multiplying $T$ by a positive constant) that $v=\mathcal{H}^{1} L J$ for some rectifiable set $J$.

Remark 3. Given a line $d$ which contains the origin, we call $\xi_{d}:=e^{i \phi}$ the unique vector in $\mathbf{S}^{1}$ which is parallel to $d$ and such that $\phi \in[0, \pi)$. Since $d$ divides $\mathbf{R}^{2}$ in two half-planes we call

upper half-plane the one which contains ${ }^{\perp} \xi_{d}$

lower half-plane the other.

If $m$ is a line-roof we will call $m_{*}^{+}$and $m_{*}^{-}$its values on the upper half-plane and the lower half-plane respectively. Hence to every line-roof, up to translations, corresponds one and only one triplet $\left(\xi_{d}, m_{*}^{+}, m_{*}^{-}\right)$. The triplet $\left(\xi, m^{+}, m^{-}\right)$associated to $m^{T}$ in Proposition 4 can be explicitly computed from $T$.

Before coming to the proof we introduce the following two lemmas: the first one is proved in [13] whereas the second one is proved in [17]. The first one will allow us to characterize jumps from their entropies and the second will be the starting point of our geometric arguments.

Lemma 3. If $\varphi \in C_{c}^{\infty}\left(\mathbf{R}^{2}\right)$ then $\Phi(z)=\varphi(z) z+\left(\nabla \varphi(z) \cdot{ }^{\perp} z\right)^{\perp} z$ is an entropy.

Lemma 4. Let us suppose that $\Omega$ is an open convex set and that $m \in A(\Omega)$ is such that for every entropy $\Phi$ we have $\mu_{\Phi}=0$. Then

(a) either there exists a point $x_{0} \in \Omega$ such that $m$ is a vortex centered at $x_{0}$ (see Definition 3(a));

(b) or $m$ is Lipschitz in every compact subset of $\Omega$.

In the second case in every point $x \in \Omega$ passes a characteristic, i.e. a line d such that $m$ is constant on $d$ and perpendicular to it. Moreover these characteristics stop only when they hit $\partial \Omega$.

Proof of Proposition 4. First Step Thanks to rectifiability we know that for $\nu$-a.e. $x$ the measures $\left\{v^{x, r} / r\right\}_{r \downarrow 0}$ converge to $g(x) \mathcal{H}^{1} L d(x)$, where $d(x)$ is a line passing through the origin and $g(x)$ a positive number. Let us call $J$ the set of these points. If $x \in J$ and $m^{\infty} \in B^{\infty}(x)$ we then have

$$
\nabla \cdot\left[\Phi\left(m^{\infty}\right)\right]=T(\Phi) g(x) \mathcal{H}^{1} L d(x) .
$$


Second Step Let $x \in J$ and $m^{\infty}$ be a given field in $B^{\infty}(x)$ and suppose, to fix ideas, that $d(x)=\{(z, 0): z \in \mathbf{R}\}$. For the sake of simplicity let us use $d$ in place of $d(x)$. From Lemma 4 (see also Lemma 3.1 of [17]) we know that on the line $d, m$ has well defined left and right trace $m^{+}, m^{-} \in L^{\infty}(d)$ (right and left is defined in the same way as in Remark 3). Thus for every interval $D \subset d$ we have

$$
\begin{aligned}
& \lim _{\varepsilon \downarrow 0} \frac{1}{\varepsilon} \int_{D \times(0, \varepsilon)}\left|m^{\infty}(z, y)-m^{+}(z)\right| d z d y=0 \\
& \lim _{\varepsilon \downarrow 0} \frac{1}{\varepsilon} \int_{D \times(-\varepsilon, 0)}\left|m^{\infty}(z, y)-m^{-}(z)\right| d z d y=0 .
\end{aligned}
$$

Now let us fix a rectangle $D \times(-\varepsilon, \varepsilon)$ : using the Fubini-Tonelli Theorem, integrating by parts (51) and letting $\varepsilon$ go to 0 we can easily see that

$$
g(x) T(\Phi) \mathcal{H}^{1}(D)=\int_{D}\left(\Phi\left(m^{+}(z)\right)-\Phi\left(m^{-}(z)\right) \cdot(1,0) d \mathcal{H}^{1}(z) .\right.
$$

This gives that $\left(\Phi\left(m^{+}\right)-\Phi\left(m^{-}\right)\right) \cdot(1,0)$ is a constant. For an $m^{\infty}$ having the line of discontinuity with direction $\xi_{d}$ we then have

$$
\left(\Phi\left(m^{+}\right)-\Phi\left(m^{-}\right)\right) \cdot{ }^{\perp} \xi_{d} \text { is constant for every entropy } \Phi .
$$

Third Step We now prove that $m^{+}$and $m^{-}$are constant. Thanks to the divergence-free constraint, $m^{+}(z)-m^{-}(z)$ is parallel to $\xi_{d}$ in $\mathcal{H}^{1}$-a.e. $z$ (from now on we will write $\xi$ for $\left.\xi_{d}\right)$. Notice also that we must have $m^{+}-m^{-} \neq 0$ a.e., otherwise we would have $\Phi\left(\mathrm{m}^{+}\right)-\Phi\left(\mathrm{m}^{-}\right)=0$ for every $\Phi$, which contradicts (51). Since $m^{ \pm}$take values in $\mathbf{S}^{1}$ we can conclude that $m^{+}(z) \cdot \xi=-m^{-}(z) \cdot \xi \neq 0$ for $\mathcal{H}^{1}$-a.e. $z$. have

Let us fix two couples $\left(m_{1}^{+}, m_{1}^{-}\right),\left(m_{2}^{+}, m_{2}^{-}\right) \in \mathbf{S}^{1} \times \mathbf{S}^{1}$ and suppose that we

$$
\begin{aligned}
& \left(m_{1}^{+}-m_{1}^{-}\right) \cdot{ }^{\perp} \xi=\left(m_{2}^{+}-m_{2}^{-}\right) \cdot{ }^{\perp} \xi=0 \\
& m_{1}^{-} \cdot \xi=-m_{1}^{+} \cdot \xi \neq 0 \\
& m_{2}^{+} \cdot \xi=-m_{1}^{2} \cdot \xi \neq 0 \\
& \left(\Phi\left(m_{1}^{+}\right)-\Phi\left(m_{1}^{-}\right)\right) \cdot{ }^{\perp} \xi=\left(\Phi\left(m_{2}^{+}\right)-\Phi\left(m_{2}^{-}\right)\right) \cdot \perp \xi \quad \forall \Phi
\end{aligned}
$$

We claim that these conditions imply $m_{1}^{+}=m_{2}^{+}, m_{1}^{-}=m_{2}^{-}$(hence from (54) we conclude that $\mathrm{m}^{+}$and $\mathrm{m}^{-}$are both constant). Arguing by contradiction we would have three possibilities:

(i) $m_{2}^{-} \neq m_{1}^{+} \neq m_{2}^{+}$

(ii) $m_{2}^{-} \neq m_{1}^{-} \neq m_{2}^{+}$;

(iii) $m_{1}^{+}=m_{2}^{-}$and $m_{1}^{-}=m_{2}^{+}$.

According to Lemma 3 if $\varphi \in C_{c}^{\infty}\left(\mathbf{R}^{2}\right)$ the map $\Phi(z)=\varphi(z) z+\left(\nabla \varphi(z) \cdot{ }^{\perp} z\right)^{\perp} z$ is an entropy. So in case (i) we choose a function $\varphi$ such that: 
$-\varphi\left(m_{i}^{ \pm}\right)=0$ for $i \in\{1,2\}$;

$-\nabla \varphi\left(m_{2}^{ \pm}\right)=0$ and $\nabla \varphi\left(m_{1}^{-}\right)=0$.

$-\nabla \varphi\left(m_{1}^{+}\right)={ }^{\perp} m_{1}^{+}$.

Then thanks to (56) we would have $\left(\Phi\left(m_{1}^{+}\right)-\Phi\left(m_{1}^{-}\right)\right) \cdot{ }^{\perp} \xi \neq 0$ and $\left(\Phi\left(m_{2}^{+}\right)-\right.$ $\left.\Phi\left(m_{2}^{-}\right)\right) \cdot{ }^{\perp} \xi=0$ which are incompatible with (58). If we were in case (ii) we could argue in a similar way (just by exchanging the roles of $m_{1}^{+}$and $m_{1}^{-}$). For handling case (iii) we choose

$-\varphi\left(m_{i}^{ \pm}\right)=0$ for $i \in\{1,2\}$;

$-\nabla \varphi\left(m_{1}^{+}\right)=\nabla \varphi\left(m_{2}^{-}\right)={ }^{\perp} m_{1}^{+}={ }^{\perp} m_{2}^{-}$;

$-\nabla \varphi\left(m_{1}^{-}\right)=\nabla \varphi\left(m_{2}^{+}\right)=0$.

Indeed such a choice would imply

$$
\left(\Phi\left(m_{1}^{+}\right)-\Phi\left(m_{1}^{-}\right)\right) \cdot{ }^{\perp} \xi=-\left(\Phi\left(m_{2}^{+}\right)-\Phi\left(m_{2}^{-}\right)\right) \cdot{ }^{\perp} \xi \neq 0 .
$$

Fourth Step Now let $x, y \in J$ and $m_{1}^{\infty} \in B^{\infty}(x), m_{2}^{\infty} \in B^{\infty}(y)$. We call $\xi_{i}$ the directions of the two lines of discontinuity and $m_{i}^{ \pm}$the right and left traces of $m_{i}^{\infty}$ on its line of discontinuity. Then equation (53) implies that

$$
\left(\Phi\left(m_{1}^{+}\right)-\Phi\left(m_{1}^{-}\right)\right) \cdot{ }^{\perp} \xi_{1}=\frac{g(y)}{g(x)}\left(\Phi\left(m_{2}^{+}\right)-\Phi\left(m_{2}^{-}\right)\right) \cdot{ }^{\perp} \xi_{2} .
$$

It is straightforward to check that if we replace (58) with $(59)$ and $(55,56,57)$ with

$$
\begin{aligned}
& \left(m_{i}^{+}-m_{i}^{-}\right) \cdot{ }^{\perp} \xi_{i}=0 \\
& m_{i}^{-} \cdot \xi_{i}=-m_{i}^{+} \cdot \xi_{i} \neq 0
\end{aligned}
$$

the proof of the previous step still works and implies $m_{1}^{+}=m_{2}^{+}$and $m_{1}^{-}=m_{2}^{-}$. As already noticed $m_{i}^{+}-m_{i}^{-}$is parallel to $\xi_{i}$. Hence the couple $\left(m_{i}^{+}, m_{i}^{-}\right)$determines $\xi_{i}$ and we can conclude that $g(x)=g(y), d(x)=d(y)$.

Thus there exists a fixed constant $c$ such that $v^{x, r} / r \stackrel{*}{\rightarrow} c \mathcal{H}^{1} L d$ for every $x \in J$. This easily implies $v=c \mathcal{H}^{1}\llcorner J$ and so our initial split-state can be characterized also with the triplet $\left(m, c T, \mathcal{H}^{1}\llcorner J)\right.$.

Fifth Step We will now end the proof by showing that for $x \in J$ every $m^{\infty} \in B^{\infty}(x)$ is constant on both the half-planes individuated by $d$. We fix our attention on the upper half-plane. We know that we have the two alternatives of Lemma 4: anyway we can rule out alternative (a), since a vortex would not give a constant trace on the line $d$. So we are in case (b) and for every point $w$ in the upper half-plane we can find a characteristic line $l_{w}$ which passes through $w$ and stops only when it hits $d$.

We notice that thanks to (52) for every $w^{\prime} \in d$ there is a sequence of points $\left\{w_{n}\right\}_{n}$ lying in the upper half-plane and such that $\left\{m^{\infty}\left(w_{n}\right)\right\}_{n}$ converges to $m^{+}$. If we take the characteristics $l_{w_{n}}$ we easily conclude that they converge (up to a subsequence) to a half-line $l_{w^{\prime}}$ which originates in $w^{\prime}$ and is perpendicular to $m^{+}$. Moreover Lipschitz continuity of $m^{\infty}$ on compact subsets of the half-planes gives that $m^{\infty}$ is constantly equal to $m^{+}$on $l_{w^{\prime}}$. This implies that $m^{\infty}$ is constantly equal to $m^{+}$ on the whole upper half-plane. 
Remark 4. We notice that Kruzkov functions are not able to distinguish every lineroof from another since, for example, the line-roofs individuated by the triplets

$$
((0,1),(a, b),(-a, b)) \quad((0,1),(a,-b),(-a,-b))
$$

have the same $f$ in equation (47). This gives a difference between the problem we are treating and the scalar one-dimensional conservation laws, in which Kruzkov's entropies alone are able to distinguish among all the "jumps".

\section{Classification of split-states}

In this section we conclude with the classification of split-states.

Proposition 5. If ( $m, T, \mathcal{H}^{1}\llcorner J)$ is a non-degenerate split-state then $m$ is a roof (either a line-roof or a half-roof or a segment-roof: see Definition 3 and compare with Fig. 1 and Fig. 7). Moreover the values $m^{+}$and $m^{-}$in Definition 3 are completely determined by $T$.

Remark 5. From Proposition 2 we know that $J$ is rectifiable. Hence without loosing our generality we may assume that in every $x \in J$ there is a line tangent to it. From Proposition 4 we know that this line is determined by $T$, i.e. is the same in every $x$. In the following we denote it by $d$. Again according to Proposition 4 , for every $x \in J, B^{\infty}(x)$ consists of a single line-roof which jumps on $d$ between two fixed values. We call these values $m_{*}^{+}$and $m_{*}^{-}$.

Before addressing the proof of Proposition 5 we need some preliminary remarks.

Remark 6. Thanks to what we have proved in the previous section we can calculate explicitly the function $f$ in terms of $m_{*}^{+}, m_{*}^{-}$and $d$. To fix ideas we suppose that $d$ is directed along $(1,0)$ and $m_{*}^{+}=(a, b)$ with $a, b>0$. Then the shape of $f$ can be easily described by Fig. 3. So we conclude that $f$ is positive on the two-sided cone $C^{+}:=A \cup C$ and negative on $C^{-}:=B \cup D$.

Lemma 5. Define $C^{+}$and $C^{-}$as in Remark 6. Then

$$
\mathcal{H}^{1}\left(\left(x+\left(C^{+} \cup C^{-}\right)\right) \cap J\right)=0 \quad \text { for every } x \in J .
$$

Proof. In view of Proposition 4 there are two sequences of Lebesgue points $\left\{x_{n}^{+}\right\}_{n \uparrow \infty}$ and $\left\{x_{n}^{-}\right\}_{n \uparrow \infty}$ both converging to $x$ such that $m\left(x_{n}^{+}\right) \rightarrow m_{*}^{+}$and $m\left(x_{n}^{-}\right) \rightarrow m_{*}^{-}$. Let us fix our attention on $x_{n}^{+}$. Since $m\left(x_{n}^{+}\right)$is close to $m_{*}^{+}$, there is a cone $A_{n}$ close to $A$ such that $\mathcal{H}^{1}\left(\left(x_{n}^{+}+A\right) \cap J\right)=0$ by the argument of Lemma 2 and Remark 6. In the limit we obtain $\mathcal{H}^{1}((x+A) \cap J)=0$, since $A$ is open. Since the proof of Lemma 2 can be adapted to the case $\chi(\xi, m(x))=0$ and $f(\xi)<0$ we obtain analogously $\mathcal{H}^{1}((x+D) \cap J)=0$. Hence $\mathcal{H}^{1}((x+(A \cup D)) \cap J)=0$. By symmetry $\mathcal{H}^{1}((x+(B \cup C)) \cap J)=0$. 


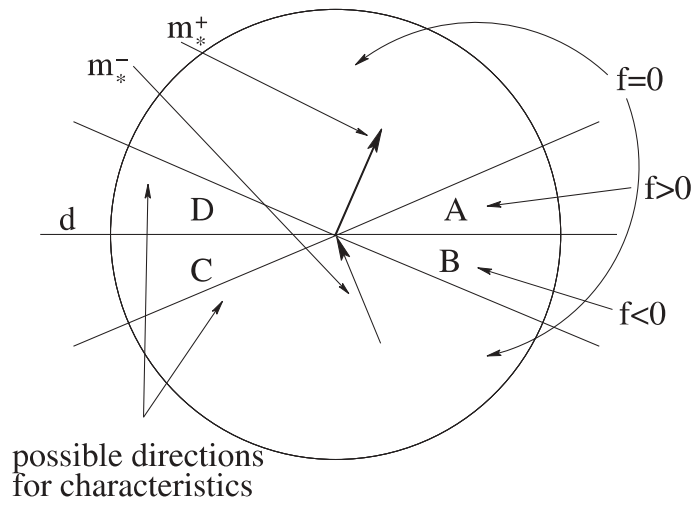

Fig. 3. The shape of $f$

Proof of Proposition 5. We divide the proof into several steps.

First Step If $x \in J$ than $\mathcal{H}^{1}((x+d) \cap J)>0$.

Let $x \in J$ be fixed and without loosing our generality suppose $x=0$. From Proposition 4 we know that the blow-up of $\mathcal{H}^{1}\left\llcorner J\right.$ in 0 is $\mathcal{H}^{1} L d$. Since the open set $C^{+} \cup C^{-} \cup d \backslash\{0\}$ contains $d \backslash\{0\}$ we have

$$
\lim _{r \downarrow 0} \frac{\mathcal{H}^{1}\left(\left(C^{+} \cup C^{-} \cup d \backslash\{0\}\right) \cap J \cap B_{r}(x)\right)}{2 r}=1
$$

and hence $\mathcal{H}^{1}\left(\left(C^{+} \cup C^{-} \cup d\right) \cap J\right)>0$. On the other hand by Lemma 5 we have $\mathcal{H}^{1}\left(\left(C^{+} \cup C^{-}\right) \cap J\right)=0$ and thus

$$
\mathcal{H}^{1}\left(\left(C^{+} \cup C^{-} \cup d\right) \cap J\right)=\mathcal{H}^{1}(d \cap J) .
$$

Second Step If $x \in J$ then $J \cap(x+d)$ is connected.

Let us suppose that $x, y \in J \cap(x+d)$ and fix a system of coordinates in which $d=\{(t, 0): t \in \mathbf{R}\}$. We know that in $x$ and $y$ every blow-up has to be a jump between $m_{*}^{+}$and $m_{*}^{-}$. To fix ideas let us suppose that $m_{*}^{-}$and $m_{*}^{+}$are oriented as in Fig. 3. Consider the half-stripe denoted by $A$ in Fig. 4: this half-stripe is bounded by the segment $[x, y]$ and by the two half-lines perpendicular to $m_{*}^{+}$which start from $x$ and $y$. Consider also the symmetric half-stripe $B$. We will prove that

$$
\begin{array}{lll}
m \equiv m_{*}^{+} & \text {on } \quad A, \\
m \equiv m_{*}^{-} & \text {on } \quad B .
\end{array}
$$

Of course this will imply $[x, y] \subset J$ and hence completes the proof of this step.

Lemma 5 ensures that $\mathcal{H}^{1}(J \cap A)=\mathcal{H}^{1}(J \cap B)=0$. Since $A$ and $B$ are convex we may apply Lemma 4 . If $m$ were a vortex on $A$ we would have the wrong trace either near $x$ or near $y$. Hence Lemma 4 implies that the characteristics drawn in the stripe stop only if they hit the boundary and that $m$ is Lipschitz on every compact subset of the stripe. 


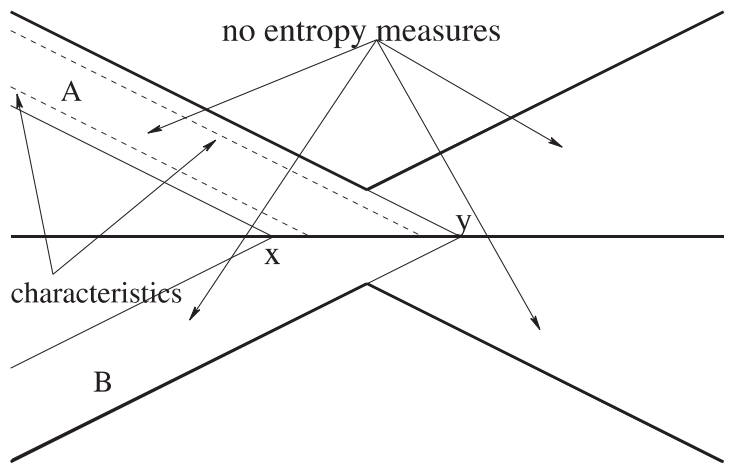

Fig. 4. The half-stripes $A$ and $B$

From the first step we know that for every $\varepsilon>0$ we can find $x^{\prime}, y^{\prime} \in J \cap d$ such that $x<x^{\prime}<y^{\prime}<y$ and $\left|x-x^{\prime}\right|,\left|y-y^{\prime}\right| \leq \varepsilon$. Reasoning exactly as in the fifth step of the proof of Proposition 4 we draw two half-lines $l_{x}^{\prime}$ and $l_{y}^{\prime}$ both perpendicular to $m_{*}^{+}$and starting respectively from $x^{\prime}$ and $y^{\prime}$. We remark that $m$ is constantly equal to $m_{*}^{+}$on them. This implies that every characteristic lying in the stripe delimited by the two half-lines $l_{x^{\prime}}$ and $l_{y^{\prime}}$ (and by $\left[x^{\prime}, y^{\prime}\right]$ ) have to be parallel to $l_{x^{\prime}}$. Hence $m \equiv m_{*}^{+}$in this stripe, and letting $x^{\prime}, y^{\prime}$ converge to $x$ and $y$ we get (61). A symmetric argument gives (62) and completes the proof.

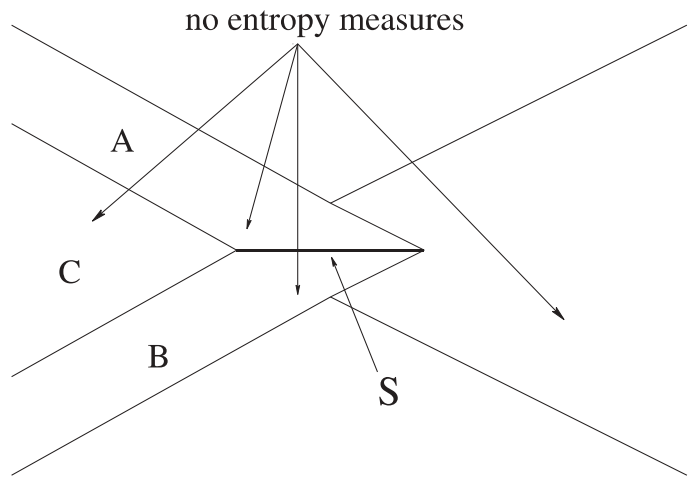

Fig. 5. Regions with no entropy measure when the segment $S$ is a connected component of $J$

Third Step $J$ is contained in one line.

The previous steps imply that $J$ is the countable union of open subsets of parallel lines. Let us suppose that $J$ is not connected. Then condition (60) implies that the connected components of $J$ are finite segments. Now, if we have a finite segment, then in the region indicated in Fig. $5 \mathcal{H}^{1}\llcorner J$ is identically 0. 
Fix one of the segments and call it $S$. In the second step we have already identified $m$ in the half-stripes $A$ and $B$. We now will identify $m$ in the cone $C$. Let $l_{1}$ and $l_{2}$ denote the characteristic half-lines which bound $C$ and $O$ its vertex, see Fig. 6. We apply Lemma 4 to $C$ and we easily conclude that $\left.m\right|_{C}$ cannot be a vortex with center in the interior of $C$. Since the characteristics lying in $C$ cannot hit $l_{1}$ or $l_{2}$ (there cannot be a vortex centered at a point of $l_{i}$ since $m$ is constant on them) they all have to hit $O$. Hence $m$ looks as in Fig. 6.

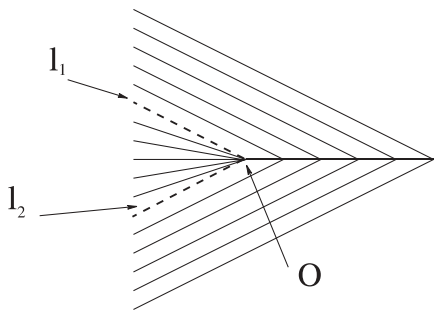

Fig. 6. Characteristics emanating from the endpoint $O$ and from the segment $S$

Finally it is easy to see that if $J$ consists of more than one segment then the characteristics emanating from the acute endpoints of the segments (which are all either the left ones or the right ones) would intersect. But this is not possible.

Fourth Step Conclusion of the proof.

From the last step we know that $J$ is either a line or a half-line or a segment. Since outside the closure of $J$ the entropy measures are identically zero, we can draw the characteristic lines as we have done in the proofs of the previous steps. It is easy to conclude that they have to be as summarized in Fig. 7 below.
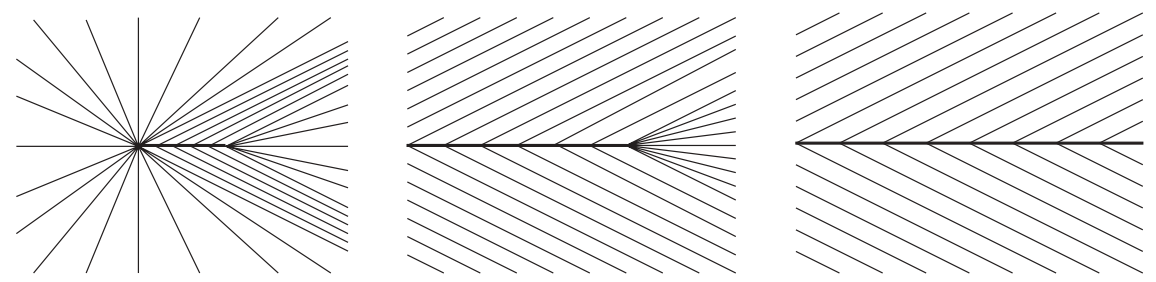

Fig. 7. The three different classes of split-states: segment-roofs, half-rofs, line-roofs

\section{Further characterization of $B^{\infty}(x)$}

In the previous sections we identified all $m^{\infty}$ 's with

$$
\nabla \cdot\left[\Phi\left(m^{\infty}\right)\right]=0 \text { for all entropies }
$$


and all split-states, that is, $m^{\infty}$ 's of the form

$$
\nabla \cdot\left[\Phi\left(m^{\infty}\right)\right]=T(\Phi) v^{\infty} \text { for all entropies. }
$$

So far, we have not made use of the fact that all these $m^{\infty}$ 's come from the blow-up of a single field in a single point. This will be done now. We obtain a complete classification of $B^{\infty}(x)$ for $x \in G \backslash J$ in Proposition 6 and for $x \in J$ in Proposition 7. Before stating them we introduce a bit of terminology.

Definition 6. A vortex will be called

- centered if its unique point of singularity is the origin;

- counterclockwise if it is of the form $m_{v}\left(x-x_{0}\right)$ (see Definition 3(a));

- clockwise if it is of the form $-m_{v}\left(x-x_{0}\right)$.

A line-roof is centered if its singular line contains the origin.

A half-roof is centered if its singular half-line starts from the origin.

Proposition 6. Let $x \in G \backslash J$ (see the proof of Proposition 1 for the definition). Then

either $B^{\infty}(x)$ contains only constants

or $B^{\infty}(x)$ contains only the centered counterclockwise vortex or $B^{\infty}(x)$ contains only the centered clockwise vortex.

Proposition 7. Let $x \in J$. Then

either $B^{\infty}(x)$ contains only the centered line-roof or $B^{\infty}(x)$ contains only the centered left half-roof or $B^{\infty}(x)$ contains only the centered right half-roof.

Proof of Proposition 6. We recall from Proposition 1 that

$$
B^{\infty}(x) \quad \text { contains only constants and vortices. }
$$

Without loosing our generality we assume that $x=0$ and that $m$ is defined everywhere in $B_{2}(0)$.

First Step A functional $\mathcal{F}$ on $m$-space.

We will define a functional $\mathcal{F}$ on all essentially bounded and weakly divergencefree vector fields $m$ on $B_{2}(0)$. Because $m$ is divergence-free, there exists a Lipschitz continuous "stream function" $\psi$ with ${ }^{\perp} \nabla \psi=m ; \psi$ is unique up to additive constants. We take $\mathcal{F}(m)$ to be an "averaged second derivative" of $\psi$ in the origin

$$
\mathcal{F}(m):=\frac{3}{2 \pi} \int_{B_{1}(0)}(\psi(x)-\psi(0)) d x .
$$

This functional is normalized such that $|\nabla \psi|^{2}=1$ a.e. implies

$$
\mathcal{F}(m) \in[-1,1] \text {. }
$$


We will be interested in how $\mathcal{F}$ behaves under rescaling. It is easy to check that

$$
\begin{aligned}
& \left.\frac{2 \pi}{3} \frac{d}{d r}\right|_{r=1}\left[\mathcal{F}\left(m_{0, r}\right)\right] \\
& =\int_{\partial B_{1}(0)}(\psi(x)-\psi(0)) d x-3 \int_{B_{1}(0)}(\psi(x)-\psi(0)) d x \\
& =\int_{\partial B_{1}(0)}\left(\psi(x)-\psi(0)-3 \int_{0}^{1}(\psi(s x)-\psi(0)) s d s\right) d x
\end{aligned}
$$

Second Step $\mathcal{F}$ separates the elements of $B^{\infty}(0)$.

We now state how $\mathcal{F}$ acts on $m^{\infty} \in B^{\infty}(0)$, i. e. on constants and vortices. We list the obvious equivalences in the following table

\begin{tabular}{|l|c|}
\hline $\mathcal{F}\left(m^{\infty}\right)=1$ & centered counterclockwise vortex \\
$\mathcal{F}\left(m^{\infty}\right) \in(0,1)$ & off-center counterclockwise vortex \\
$\mathcal{F}\left(m^{\infty}\right)=0$ & constant \\
$\mathcal{F}\left(m^{\infty}\right) \in(-1,0)$ & off-center clockwise vortex \\
$\mathcal{F}\left(m^{\infty}\right)=-1$ & centered clockwise vortex \\
\hline
\end{tabular}

We next state how $\mathcal{F}$ acts on rescaled versions $m_{0, r}^{\infty}$ of $m^{\infty} \in B^{\infty}(0)$ :

$$
\begin{gathered}
\left.\mathcal{F}\left(m^{\infty}\right) \in(0,1) \Longrightarrow \frac{d}{d r}\right|_{r=1}\left[\mathcal{F}\left(m_{0, r}^{\infty}\right)\right]>0, \\
\left.\mathcal{F}\left(m^{\infty}\right) \in(-1,0) \Longrightarrow \frac{d}{d r}\right|_{r=1}\left[\mathcal{F}\left(m_{0, r}^{\infty}\right)\right]<0 .
\end{gathered}
$$

Let us now argue in favor of, say, (65). In view of the table, $\mathcal{F}\left(m^{\infty}\right) \in(0,1)$ implies that $m^{\infty}$ is an off-center counterclockwise vortex. In particular, the related stream function $\psi^{\infty}$ is convex and we have

$$
\psi^{\infty}(r x)-\psi^{\infty}(0) \leq\left(\psi^{\infty}(x)-\psi^{\infty}(0)\right) r \quad \text { for } x \in \partial B_{1} \text { and } r \in[0,1] .
$$

From the last line in (64) we see that $\left.\frac{d}{d r}\right|_{r=1}\left[\mathcal{F}\left(m_{0, r}^{\infty}\right)\right]$ is always nonnegative, and vanishes only if $\psi^{\infty}$ is homogeneous of degree 1 in $B_{1}(0)$. But this is not the case for an off-center vortex. Hence (65) holds.

Third Step Compactness argument.

We set for convenience

$$
f(r):=\mathcal{F}\left(m_{0, r}\right)=\frac{3}{2 \pi} \int_{B_{1}(0)}\left(\psi_{0, r}(x)-\psi_{0, r}(0)\right) d x
$$

and observe that (see (64))

$$
\begin{aligned}
\frac{2 \pi}{3} r f^{\prime}(r)= & \int_{\partial B_{1}(0)}\left(\psi_{0, r}(x)-\psi_{0, r}(0)\right) d x \\
& -3 \int_{B_{1}(0)}\left(\psi_{0, r}(x)-\psi_{0, r}(0)\right) d x
\end{aligned}
$$


where $\psi_{0, r}(x)-\psi_{0, r}(0)=\frac{1}{r}(\psi(r x)-\psi(0))$ is the stream function for $m_{0, r}$. We claim that for all $\delta>0$, there exist $\varepsilon>0, r_{0}>0$ s.t.

$$
\begin{aligned}
f(r) \in[\delta, 1-\delta] \text { and } r & \leq r_{0} \Longrightarrow r f^{\prime}(r)>\varepsilon, \\
f(r) \in[-1+\delta,-\delta] \text { and } r \leq r_{0} \Longrightarrow r f^{\prime}(r) & <-\varepsilon .
\end{aligned}
$$

We reason by contradiction in favor of (67) and assume that there exists a sequence $\left\{r_{n}\right\}_{n}$ converging to zero such that

$$
\lim _{n \rightarrow \infty} f\left(r_{n}\right) \in(0,1) \text { and } \lim _{n \rightarrow \infty} r_{n} f^{\prime}\left(r_{n}\right) \leq 0
$$

We may also assume that $\left\{m_{0, r_{n}}\right\}_{n}$ converges strongly to an $m^{\infty} \in B^{\infty}(0)$, which implies uniform convergence of $\left\{\psi_{0, r_{n}}\right\}_{n}$ to the corresponding $\psi^{\infty}$. Hence we obtain

$$
\begin{array}{r}
\mathcal{F}\left(m^{\infty}\right)=\lim _{n \rightarrow \infty} f\left(r_{n}\right) \stackrel{(68)}{\in}(0,1), \\
\left.\frac{d}{d r}\right|_{r=1}\left[\mathcal{F}\left(m_{0, r}^{\infty}\right)\right] \stackrel{(64,66)}{=} \lim _{n \rightarrow \infty} r_{n} f^{\prime}\left(r_{n}\right) \stackrel{(68)}{\leq} 0 .
\end{array}
$$

This is a contradiction according to (65).

Fourth Step Ode argument.

Since $\int_{0}^{r_{0}} \frac{1}{r} d r=\infty,(67)$ implies

$$
\liminf _{r \downarrow 0} f(r)<1-\delta \Longrightarrow \limsup _{r \downarrow 0} f(r) \leq \delta .
$$

Indeed, it is obvious from (67) that

$$
\begin{aligned}
& \liminf _{r \downarrow 0} f(r) \leq \delta \Longrightarrow \limsup _{r \downarrow 0} f(r) \leq \delta \\
& \liminf _{r \downarrow 0} f(r) \leq 1-\delta \Longrightarrow \limsup _{r \downarrow 0} f(r) \leq 1-\delta \text {. }
\end{aligned}
$$

We conclude from (70) that for proving (69) it is sufficient to show

$$
\liminf _{r \downarrow 0} f(r) \leq \delta .
$$

We argue by contradiction: if this is false then for some $\rho_{1}$ we have $f(r) \in[\delta,+\infty[$ for any $\left.r \in] 0, \rho_{1}\right]$. But, taking into account (71) and the left-hand side of (69) we also conclude that there exists $\rho_{2}$ such that $\left.f(r) \in\right]-\infty, 1-\delta$ ] for any $\left.\left.r \in\right] 0, \rho_{2}\right]$. So if $\rho=\min \left\{\rho_{1}, \rho_{2}\right\}$ we would have $f(r) \in[\delta, 1-\delta]$ for all $\left.\left.r \in\right] 0, \rho\right]$ and therefore

$$
+\infty>\limsup _{t \downarrow 0}(f(\rho)-f(t)) \geq \limsup _{t \downarrow 0} \int_{t}^{\rho} f^{\prime}(r) d r \stackrel{(67)}{\geq} \varepsilon \int_{0}^{\rho} \frac{d r}{r}=+\infty,
$$

which is a contradiction. 
Since $\delta>0$ was arbitrary (69) yields

$$
\liminf _{r \downarrow 0} f(r)<1 \Longrightarrow \limsup _{r \downarrow 0} f(r) \leq 0 .
$$

By symmetry, we also have

$$
\underset{r \downarrow 0}{\limsup } f(r)>-1 \Longrightarrow \liminf _{r \downarrow 0} f(r) \geq 0 .
$$

Hence in view of (63), we only have three cases:

$$
\lim _{r \downarrow 0} f(r)=0 \quad \text { or } \quad \lim _{r \downarrow 0} f(r)=1 \quad \text { or } \quad \lim _{r \downarrow 0} f(r)=-1 \text {. }
$$

Hence $\mathcal{F}\left(B^{\infty}(0)\right)$ either is $\{0\},\{1\}$, or $\{-1\}$. In view of the above table, this implies the claim of the lemma.

Proof of Proposition 7. We recall that from Proposition 5 we know already

$$
\begin{aligned}
& B^{\infty}(x) \text { contains only constants, vortices and roofs } \\
& B^{\infty}(x) \text { contains at least one roof } \\
& \text { all roofs in } B^{\infty}(x) \text { have same triplet }\left(\xi, m^{+}, m^{-}\right) .
\end{aligned}
$$

This last statement means that

- the direction of the set of discontinuity (which is a connected piece of a line) is determined by a vector $\xi$ not depending on $m$;

- on this set any $m \in B^{\infty}(x)$ jumps between two given values $m^{-}$and $m^{+}$(and only one possibility is given, i.e. fixed $\xi$ as in Remark 3, $m$ jumps from $m^{-}$to $m^{+}$along ${ }^{\perp} \xi$, while it cannot jump from $m^{+}$to $m^{-}$).

By a change of coordinates, we may without loosing our generality assume $x=0$ and $\xi=(1,0)$. Also, possibly passing to $-m$ instead of $m$, we may assume that

$$
\text { all roofs in } B^{\infty}(x) \text { are convex }
$$

and, rescaling if necessary, that $m$ is defined in all of $B_{2}(0)$.

First Step The functional $\mathcal{F}$.

As in Proposition 6, $\psi$ denotes the stream function of $m:{ }^{\perp} \nabla \psi=m$. Let $v^{+}, v^{-}$ denote the vectors with ${ }^{\perp} v^{ \pm}=m^{ \pm}$. Hence $v^{ \pm}$are the singled-out values of $\nabla \psi$ in the line-roof case. We consider

$$
\mathcal{F}(m):=\int_{0}^{1}\left(\psi\left(s v^{+}\right)+\psi\left(s v^{-}\right)-2 \psi(0)\right) d s
$$


and observe that

$$
\begin{aligned}
& \left.\frac{d}{d r}\right|_{r=1}\left[\mathcal{F}\left(m_{0, r}\right)\right] \\
& =\psi\left(v^{+}\right)+\psi\left(v^{-}\right)-2 \psi(0)-2 \int_{0}^{1}\left(\psi\left(s v^{+}\right)+\psi\left(s v^{-}\right)-2 \psi(0)\right) d s \\
& =\psi\left(v^{+}\right)-\psi(0)-2 \int_{0}^{1}\left(\psi\left(s v^{+}\right)-\psi(0)\right) d s \\
& \quad+\psi\left(v^{-}\right)-\psi(0)-2 \int_{0}^{1}\left(\psi\left(s v^{-}\right)-\psi(0)\right) d s
\end{aligned}
$$

Second Step How functional $\mathcal{F}$ acts on $B^{\infty}(0)$

Let $m^{\infty} \in B^{\infty}(0)$ be given. It follows immediately from $|\nabla \psi|^{2}=1$ a.e. that

$$
\mathcal{F}\left(m^{\infty}\right) \in[-1,1] .
$$

In view of (72) we have

$$
\mathcal{F}\left(m^{\infty}\right)\left\{\begin{array}{l}
\geq \\
\leq
\end{array}\right\} 0 \Longrightarrow \psi^{\infty}\left\{\begin{array}{c}
\text { convex } \\
\text { concave }
\end{array}\right\} .
$$

The directions $v^{+}, v^{-}$are just chosen such that $\mathcal{F}\left(m^{\infty}\right)=1$ for roofs $m^{\infty}$ whose singular set (ridge) contains 0 . Of course $\mathcal{F}\left(m^{\infty}\right)= \pm 1$ is also true for a vortex $m^{\infty}$ with singular set (center) in 0 . Hence in view of (72) we have

$$
0 \in \text { singular set } \Longrightarrow \mathcal{F}\left(m^{\infty}\right) \in\{-1,1\}
$$

The converse statement

$$
\mathcal{F}\left(m^{\infty}\right) \in\{-1,1\} \quad \Longrightarrow \quad 0 \in \text { singular set }
$$

is also true: since $|\nabla \psi|^{2}=1$ a.e. , $\mathcal{F}\left(m^{\infty}\right) \in\{-1,1\}$ implies that $\psi^{\infty}$ is affine with slope one along the segments $[0,1] v^{+}$and $[0,1] v^{-}$. Assume that $0 \notin$ singular set. Then $\nabla \psi^{\infty}(0)$ exists so that the above translates into $\nabla \psi^{\infty}(0) \cdot v^{+}=$ $\nabla \psi^{\infty}(0) \cdot v^{-}=1$. Since $v^{+} \neq v^{-}$, this yields $\left|\nabla \psi^{\infty}(0)\right|^{2}>1-$ a contradiction. This establishes (79).

We now observe that there exists a $c_{0} \in(0,1)$ such that

$$
\psi^{\infty} \text { linear } \Longrightarrow\left|\mathcal{F}\left(m^{\infty}\right)\right| \leq c_{0} .
$$

Indeed, if $\psi^{\infty}$ is linear, we have $\mathcal{F}\left(m^{\infty}\right)=\frac{1}{2}\left(v^{+}+v^{-}\right) \cdot \nabla \psi^{\infty}(0)$ and thus $\left|\mathcal{F}\left(m^{\infty}\right)\right| \leq\left|\frac{1}{2}\left(v^{+}+v^{-}\right)\right|=: c_{0}$ because of $\left|\nabla \psi^{\infty}(0)\right|^{2}=1$. Observe that $c_{0}<1$ because of $v^{+} \neq v^{-}$. This establishes (80). Finally, we have that

$$
\begin{gathered}
\left.\mathcal{F}\left(m^{\infty}\right) \in\left(c_{0}, 1\right) \Longrightarrow \frac{d}{d r}\right|_{r=1}\left[\mathcal{F}\left(m_{0, r}^{\infty}\right)\right]>0, \\
\left.\mathcal{F}\left(m^{\infty}\right) \in\left(-1,-c_{0}\right) \Longrightarrow \frac{d}{d r}\right|_{r=1}\left[\mathcal{F}\left(m_{0, r}^{\infty}\right)\right]<0 .
\end{gathered}
$$


Let us argue in favor of, say, (81). Since in particular $\mathcal{F}\left(m^{\infty}\right) \geq 0$ we have that $\psi^{\infty}$ is convex according to (77). Looking at (75), one realizes that as in the proof of Proposition 6, the convexity implies that $\left.\frac{d}{d r}\right|_{r=1}\left[\mathcal{F}\left(m_{0, r}^{\infty}\right)\right] \geq 0$. It also shows that equality, which we shall assume, can only occur if $\psi^{\infty}$ is affine along $[0,1] v^{+}$ and $[0,1] v^{-}$. Since $\left|\mathcal{F}\left(m^{\infty}\right)\right|<1,0$ is not in the singular set according to (78). Hence $\nabla \psi^{\infty}(0)$ exists and thus $\psi^{\infty}$ is affine on the union $[0,1] v^{+} \cup[0,1] v^{-}$ of the two segments. Since $\mathcal{F}\left(m^{\infty}\right)$ only depends on the restriction of $\psi^{\infty}$ onto $[0,1] v^{+} \cup[0,1] v^{-}$, we may apply (80), which yields $\left|\mathcal{F}\left(m^{\infty}\right)\right| \leq c_{0}$ - the desired contradiction.

Third Step Conclusions from functional $\mathcal{F}$

From $(76,81,82)$, we obtain, by the same argument as in the proof of Proposition 6 , that

$$
\mathcal{F}\left(B^{\infty}(0)\right)=\{1\}, \quad \mathcal{F}\left(B^{\infty}(0)\right)=\{-1\} \text { or } \mathcal{F}\left(B^{\infty}(0)\right) \subset\left[-c_{0}, c_{0}\right] .
$$

The second case, i.e. $\mathcal{F}\left(B^{\infty}(0)\right)=\{-1\}$, is easily ruled out: according to (77) this implies that for any $m^{\infty} \in B^{\infty}(0) \quad \psi^{\infty}$ is concave and according to (79) it implies that 0 is in its singular set. In view of $(72,74)$ this means that any $m^{\infty} \in B^{\infty}(x)$ is a vortex, which contradicts (73).

Also the third case, i.e. $\mathcal{F}\left(B^{\infty}(0)\right) \subset\left[-c_{0}, c_{0}\right]$, can be ruled out: according to (73) there exists an $m^{\infty} \in B^{\infty}(0)$ with non-empty singular set. Consider its rescaled versions and their "blow-down" $m^{\infty,-\infty}$ :

$$
m_{0, r}^{\infty} \stackrel{r \uparrow \infty}{\longrightarrow} m^{\infty,-\infty} .
$$

A diagonal argument shows that also $m^{\infty,-\infty} \in B^{\infty}(0)$. On the other hand, 0 must be in the singular set of $m^{\infty,-\infty}$ so that $\left|\mathcal{F}\left(m^{\infty,-\infty}\right)\right|=1$ in view of (78) - which is in contradiction with $\mathcal{F}\left(B^{\infty}(0)\right) \subset\left[-c_{0}, c_{0}\right]$.

Hence we must have $\mathcal{F}\left(B^{\infty}(0)\right)=\{1\}$ which in view of (79), (77) and (74) translates into

$$
\begin{gathered}
B^{\infty}(0) \text { contains only the centered counterclockwise vortex } \\
\text { or roofs whose singular set runs through } 0 \text {. }
\end{gathered}
$$

Fourth Step The functional $\mathcal{G}$

In order to further restrict $B^{\infty}(0)$, we introduce

$$
\mathcal{G}(m):=2 \int_{0}^{1}(\psi(s(1,0))-\psi(0)) d s
$$

and notice that

$$
\begin{aligned}
& \left.\frac{d}{d r}\right|_{r=1}\left[\mathcal{G}\left(m_{0, r}\right)\right] \\
& =2\left(\psi((1,0))-\psi(0)-2 \int_{0}^{1}(\psi(s(1,0))-\psi(0)) d s\right) .
\end{aligned}
$$


Fifth Step How $\mathcal{G}$ acts on $B^{\infty}(0)$.

Let $m^{\infty} \in B^{\infty}(0)$ be given. We now list the properties which immediately follow from (83)

$$
\begin{aligned}
\mathcal{G}\left(m^{\infty}\right) \in[0,1], & \\
\mathcal{G}\left(m^{\infty}\right)=1 \Longrightarrow & m^{\infty} \text { is the centered counterclockwise vortex } \\
& \text { or the centered left half-roof, } \\
\mathcal{G}\left(m^{\infty}\right)=0 \Longrightarrow & m^{\infty} \text { is the centered line-roof } \\
& \text { or the centered right half-roof. }
\end{aligned}
$$

We also have

$$
\left.\mathcal{G}\left(m^{\infty}\right) \in(0,1) \Longrightarrow \frac{d}{d r}\right|_{r=1}\left[\mathcal{G}\left(m_{0, r}^{\infty}\right)\right]>0 .
$$

Indeed, consider (84): The convexity of $\psi^{\infty}$ (guaranteed by (83)), implies as in Proposition 6 that $\left.\frac{d}{d r}\right|_{r=1}\left[\mathcal{G}\left(m_{0, r}^{\infty}\right)\right] \geq 0$ with equality only if $\psi^{\infty}$ is affine on the segment $[0,1](1,0)$. In view of $(83)$, affinity would imply $\psi^{\infty}((1,0))-\psi^{\infty}(0) \in$ $\{0,1\}$ and therefore $\mathcal{G}\left(m^{\infty}\right) \in\{0,1\}$ - a contradiction.

Sixth Step Conclusions from functional $\mathcal{G}$

Again, we apply the argument from Proposition 6 and obtain from $(85,88)$ that

$$
\mathcal{G}\left(B^{\infty}(0)\right)=\{1\} \quad \text { or } \mathcal{G}\left(B^{\infty}(0)\right)=\{0\} .
$$

In view of $(86,87)$, this means

either $B^{\infty}(0) \subset$ \{centered c.c. vortex, centered left half-roof

or $B^{\infty}(0) \subset$ \{centered line-roof, centered right half-roof .

By symmetry, we also have

either $B^{\infty}(0) \subset$ \{centered c.c. vortex, centered right half-roof

or $B^{\infty}(0) \subset$ \{centered line-roof, centered left half-roof\}.

The combination of both yields that $B^{\infty}(0)$ either consists of the centered counterclockwise vortex, or the centered left half-roof, or the centered line-roof, or the centered right half-roof. Since the first case is not an option in view of (73), we obtain the claim of the proposition.

\section{Rectifiability}

In this section we will prove that the set $J$ defined in Proposition 1 satisfies the requirements of Theorem 1 . We recall that (9) has already been proved as point (d) of Proposition 1. 


\section{Proposition 8.}

(a) $J$ is rectifiable.

(b) For $\mathcal{H}^{1}$-a.e. $x \in J$ there exist $m^{+}(x)$ and $m^{-}(x)$ such that

$$
\begin{aligned}
& \lim _{r \downarrow 0} \frac{1}{r^{2}}\left\{\int_{B_{r}^{+}(x)}\left|m(y)-m^{+}(x)\right| d y\right. \\
& \left.+\int_{B_{r}^{-}(x)}\left|m(y)-m^{-}(x)\right| d y\right\}=0 .
\end{aligned}
$$

(c) For $\mathcal{H}^{1}$-a.e. $x \notin J$ we have

$$
\lim _{r \downarrow 0} \frac{1}{r^{2}} \int_{B_{r}(x)}\left|m(y)-\bar{m}_{r}\right| d y=0 .
$$

(d) If $\Phi$ is an entropy, then

$$
\mu_{\Phi}\left\llcorner J=\left[\eta \cdot\left(\Phi\left(m^{+}\right)-\Phi\left(m^{-}\right)\right)\right] \mathcal{H}^{1}\llcorner J .\right.
$$

Proof. First Step Proof of (a).

Let $\Phi$ be an entropy. Relation (21) and point (d) of Proposition 1 imply that there exists a Borel function $g_{\Phi}$ such that $\mu_{\Phi}=g_{\Phi} \mathcal{H}^{1} L J+\tilde{\mu}$, where $\tilde{\mu}(H)=0$ if $\mathcal{H}^{1}(H)<\infty$. Hence if we define $v:=g_{\Phi} \mathcal{H}^{1}\llcorner J$ we have

$$
\frac{\mu_{\Phi}^{x, r}-v^{x, r}}{r} \stackrel{*}{\rightarrow} 0 \quad \text { for } \mathcal{H}^{1} \text {-a.e. } x \in J .
$$

Proposition 7 implies that

$$
\frac{\mu_{\Phi}^{x, r}}{r} \stackrel{*}{\rightarrow} c(x) \mathcal{H}^{1} \operatorname{L} l(x) \quad \text { for } \mathcal{H}^{1} \text {-a.e. } x \in J
$$

where $c(x)$ is a real number and $l(x)$ is either a line which contains the origin or a half-line emanating from the origin.

Standard arguments imply that $\|\nu\|=\left|g_{\Phi}\right| \mathcal{H}^{1} L J$ and hence reasoning as in the Sixth Step of the proof of Lemma 1 we conclude that

$$
\frac{\|v\|^{x, r}-\operatorname{sign}\left(g_{\Phi}(x)\right) v^{x, r}}{r} \stackrel{*}{\rightarrow} 0
$$

in every $x$ which is a Lebesgue point for $g_{\Phi}$ with respect to $\mathcal{H}^{1}\llcorner J$.

Hence (92), (91) and (93) give

$$
\frac{\|v\|^{x, r}}{r} \stackrel{*}{\rightarrow}|c(x)| \mathcal{H}^{1}\llcorner l(x) \quad \text { for }\|v\|-\text { a.e. } x .
$$

Since $\|v\|$ is a nonnegative measure this implies that

$$
\liminf _{r \rightarrow 0} \frac{\|v\|\left(B_{r}(x)\right)}{r}=\limsup _{r \rightarrow 0} \frac{\|v\|\left(B_{r}(x)\right)}{r} \quad \text { for }\|v\|-\text { a.e. } x
$$


and that for $\|v\|$-a.e. $x$ there exists a cone $C_{\eta(x)}:=\{v: 2|v \cdot \eta(x)| \geq|v|\}$ such that

$$
\limsup _{r \rightarrow 0} \frac{\|\nu\|\left(\left(x+C_{\eta(x)}\right) \cap B_{r}(x)\right)}{r}=0 .
$$

Then it is a standard fact (see for example the Proof of Theorem 2.83 in [3]) that $\|v\|$ is rectifiable (actually $\|v\|=\left|g_{\Phi}\right| \mathcal{H}^{1}\llcorner J$ and (96) are already sufficient for rectifiability: see Corollary 15.16 in [23]).

Hence we have that $\left\{g_{\Phi} \neq 0\right\} \cap J$ is a rectifiable set for any entropy $\Phi$. Now recall the set of entropies $\mathcal{C}$ introduced in Warning 1. According to (36) and (28) we have

$$
J \subset \bigcup_{\Phi \in \mathcal{C}}\left\{x: \limsup _{r \rightarrow 0} \frac{\left\|\mu_{\Phi}\right\|\left(B_{r}(x)\right)}{r}>0\right\} .
$$

Hence we conclude

$$
\mathcal{H}^{1}\left(\left(\bigcup_{\Phi \in \mathcal{C}}\left\{g_{\Phi} \neq 0\right\}\right) \backslash J\right)=0,
$$

which proves the rectifiability of $J$.

Second Step Proof of (b).

Proposition 7 implies that, for $\mathcal{H}^{1}$-a.e. $x \in J, B^{\infty}(x)$ consists either of a single line-roof or of a single half-line roof. But thanks to the rectifiability of $J$ and to (97), for $\mathcal{H}^{1}$-a.e. $x \in J$ we can find an entropy $\Phi$ such that

$$
\frac{\mu_{\Phi}^{x, r}}{r} \stackrel{*}{\rightarrow} g_{\Phi}(x) \mathcal{H}^{1}\llcorner d(x)
$$

where $g_{\Phi}(x) \neq 0$ and $d(x)$ is the tangent line to $J$ in $x$. In such an $x, B^{\infty}(x)$ must then consist of a line-roof which jumps on $d(x)$. This easily gives (89).

Third Step Proof of (c).

We know from Proposition 6 that, for $\mathcal{H}^{1}$-a.e. $x \notin J$, either $B^{\infty}(x)$ consists of constants, or it consists of a single centered vortex. If $B^{\infty}(x)$ contains only constants then for every sequence $r_{n} \downarrow 0$ we can extract a subsequence $r_{h(n)}$ such that

$$
\lim _{n \rightarrow \infty} \frac{1}{r_{h(n)}^{2}} \int_{B_{r_{h(n)}}(x)}\left|m(y)-\bar{m}_{r_{h(n)}}\right| d y=0 .
$$

Thus in this case $x$ satisfies (90). We will complete the proof by showing that

$$
V:=\left\{x \mid B^{\infty}(x) \text { consists of the centered counterclockwise vortex }\right\}
$$

is countable (the same holds for the clockwise vortex). Let $m^{x}$ denote the counterclockwise vortex centered at $x$ and define

$$
\delta:=\int_{B_{1}(x) \cap B_{1}(y)}\left|m^{x}(z)-m^{y}(z)\right| d z \quad \text { for }|x-y|=1 .
$$


A scaling argument yields

$$
\int_{B_{\rho}(x) \cap B_{\rho}(y)}\left|m^{x}(z)-m^{y}(z)\right| d z=\delta \rho^{2} \quad \text { for }|x-y|=\rho
$$

Hence the set

$$
V_{r}:=\left\{x: \int_{B_{\rho}(x)}\left|m(z)-m^{x}(z)\right| d z<\frac{\delta \rho^{2}}{2} \text { for every } \rho \leq r\right\}
$$

is at most countable, because for $x \neq y \in V_{r}$ we have $|x-y|>r$ by (98) and the triangle inequality. On the other hand we have by definition

$$
V \subset \bigcup_{i=1}^{\infty} V_{\frac{1}{i}}
$$

so that also $V$ is countable.

Fourth Step Proof of (d).

Let $\Phi$ be a given entropy. By (b) we know that, for $\mathcal{H}^{1}$-a.e. $x \in J, B^{\infty}(x)$ consists of a line roof $m_{x}^{\infty}$, jumping between the two values $m^{+}(x)$ and $m^{-}(x)$ on the tangent line $d(x)$ to $J$ in $x$. Hence for these $x$ we have

$$
m_{x, r} \rightarrow m_{x}^{\infty} \quad \text { strongly in } L_{l o c}^{p} \text { for } p<\infty
$$

and thus

$$
\frac{\mu_{\Phi}^{x, r}}{r} \stackrel{*}{\rightarrow}\left[\eta(x) \cdot\left(\Phi\left(m^{+}(x)\right)-\Phi\left(m^{-}(x)\right)\right] \mathcal{H}^{1}\llcorner d(x),\right.
$$

where $\eta(x)$ is the unit normal to $J$ in $x$ such that (89) holds.

We recall again that thanks to relation (21) and point (d) of Proposition 1 we have $\mu_{\Phi}\left\llcorner J=g_{\Phi}\left\llcorner J+\tilde{\mu}\right.\right.$ where $\tilde{\mu}(H)=0$ if $\mathcal{H}^{1}(H)<\infty$. Hence we have

$$
\frac{\mu_{\Phi}^{x, r}}{r} \stackrel{*}{\rightarrow} g_{\Phi}(x) \mathcal{H}^{1} L d(x) \quad \text { for } \mathcal{H}^{1} \text {-a.e. } x \in J .
$$

Comparing (100) with (99) we conclude that $g_{\Phi}(x)=\eta(x) \cdot\left(\Phi\left(m^{+}(x)\right)-\right.$ $\Phi\left(m^{-}(x)\right)$ for $\mathcal{H}^{1}$-a.e. $x \in J$. This completes the proof.

\section{Final remarks}

In this last section we show the fact which encouraged us to come with the arguments used in Sect. 7 and we also explain why the classification of Sect. 6 is still not sufficient for proving directly the rectifiability of $J$.

Bernd Kirchheim pointed out to us that the following result holds:

Theorem 2. Let $v=g \mathcal{H}^{1} L S$ for some $S$ such that $\mathcal{H}^{1}(S)<\infty$ and moreover suppose that for $v$-a.e. $x$ the weak limits of sequences of rescaled measures $v^{x, r_{n}} / r_{n}$ with $r_{n} \downarrow 0$ can only be 
(a) a constant $c(x)$ times the Hausdorff measure concentrated on a line parallel to a fixed one $(d(x))$;

(b) the zero measure.

Then $S$ is rectifiable.

See [4] for a proof. The core of the argument is the fact that the measure $\mathcal{H}^{1}$ restricted to a line $l$ is a monotone measure, i.e. for every $x$ and every $r<s$

$$
\frac{\mathcal{H}^{1}\left(l \cap B_{r}(x)\right)}{r} \leq \frac{\mathcal{H}^{1}\left(l \cap B_{s}(x)\right)}{s},
$$

where the equality holds if and only if $x \in l$.

Using known results in Geometric Measure Theory one can extend the previous theorem substituting (a) with

(a') a constant $c>k(x)>0$ times the Hausdorff measure concentrated on some line $d$, where both $c$ and $d$ may depend freely on the chosen sequence $\left\{r_{n}\right\}$, whereas $k(x)$ does not depend on it.

Also, see again [4], one could allow for a third possibility for the limits of rescaled measures, namely

(c) a constant $c$ times the Hausdorff measure concentrated on some half-line $d$ (where, as in (a'), $d$ is allowed to vary among all half-lines and c is only subject to the constraint $c>k(x)>0)$.

Anyway it is not clear to us if the same could hold allowing the rescaled measures to possibly converge also to segments. We are here very near to a borderline between rectifiability and unrectifiability. Indeed let us take the measure $v$ given by the restriction of $\mathcal{H}^{1}$ to the graph of the function

$$
f(x):=\sum_{i=1}^{\infty} 2^{-n^{2}} \chi_{A_{n}}(x),
$$

being $A_{n}$ the union of the closed segments $\left[\left(k-2^{-[\log n]}\right) 2^{-n^{2}}, k 2^{-n^{2}}\right]$, where $k \in\left\{1,2, \ldots, 2^{n^{2}}\right\}$ and $[x]$ denotes the integer part of $x$. This example is a slight modification of one shown by Dickinson in [14] and it can be proved that

(i) $\quad v$ is not rectifiable (i.e. the graph of $f$ is an unrectifiable set);

(ii) for $v$-a.e. $x$, if the measures $v^{x, r_{n}} / r_{n}$ converge then their weak limit is given by $\mathcal{H}^{1}$ restricted to a subset $J$ of $\{(x, y), x \in \mathbf{R}\}$ for some $y \in \mathbf{R}$ (where $y$ depends on the sequence $\left\{r_{n}\right\}$ );

(iii) the subsets $J$ which appear in (ii) consist all of at most two connected components (more precisely they can be the full line, an half-line, a segment, the full line minus a segment or the empty set).

The proofs of these facts are just a routine modification of those present in [14]. 


\section{References}

1. Alouges, F., Rivière, T., Serfaty, S.: Wall energies of micromagnetic materials with strong planar anisotropy. ESAIM, Control Optim. Calc. Var. 8, 31-68 (2002)

2. Ambrosio, L., De Lellis, C., Mantegazza, C.: Line energies for gradient vector fields in the plane. Calc. Var. Partial Differ. Equ. 9, 327-355 (1999)

3. Ambrosio, L., Fusco, N., Pallara, D.: Functions of bounded variation and free discontinuity problems. Oxford Mathematical Monographs. Oxford: Clarendon Press 2000

4. Ambrosio, L., Kirchheim, B., Lecumberry, M., Rivière, T.: The local structure of Néel Walls. To appear in the Volume dedicated to the 80th birthday of O. Ladyzhenskaya. Kluwert Academic 2002

5. Ambrosio, L., Lecumberry, M., Rivière, T.: Viscosity property of minimizing micromagnetic configurations. To appear in Commun. Pure Appl. Math.

6. Aviles, P., Giga, Y.: A mathematical problem related to the physical theory of liquid crystals configurations. Proc. Centre Math. Anal. Austral. Nat. Univ. 12, 1-16 (1987)

7. Aviles, P., Giga, Y.: On lower semicontinuity of a defect energy obtained by a singular limit of the Ginzburg-Landau type energy for gradient fields. Proc. R. Soc. Edinb., Sect. A, Math. 129, 1-17 (1999)

8. Ben Belgacem, H., Conti, S., De Simone, A., Müller, S.: Energy scaling of compressed elastic films: three-dimensional elasticity and reduced theories. Arch. Ration. Mech. Anal. 164, 1-37 (2002)

9. De Lellis, C.: Energie di linea per campi di gradienti. Ba. D. Thesis. University of Pisa 1999

10. De Lellis, C.: An example in the gradient theory of phase transitions. ESAIM, Control Optim. Calc. Var. 7, 285-289 (2002)

11. De Lellis, C., Otto, F., Westdickenberg, M.: Structure of entropy solutions for multidimensional scalar conservation laws.

Preprint Nr. 95/2002 at http://www.mis.mpg.de/preprints

12. De Simone, A., Kohn, R.V., Müller, S., Otto, F.: A reduced theory for thin-film micromagnetics. Commun. Pure Appl. Math. 55, 1408-1460 (2002)

13. De Simone, A., Kohn, R.V., Müller, S., Otto, F.: A compactness result in the gradient theory of phase transition. Proc. R. Soc. Edinb., Sect. A, Math. 131, 833-844 (2001)

14. Dickinson, D.R.: Study or extreme cases with respect to densities of irregular linearly measurable plane sets of points. Math. Ann. 116, 358-373 (1939)

15. Di Perna, R., Lions, P.L., Meyer, Y.: $L^{p}$ regularity of velocity averages. Ann. Inst. Henri Poincaré, Anal. Non Linéaire 8, 271-287 (1991)

16. Federer, H.: Geometric measure theory. Classics in Mathematics. Berlin, Heidelberg, New York: Springer 1969

17. Jabin, P.E., Otto, F., Perthame, B.: Line-energy Ginzburg-Landau models: zero-energy states. Ann. Sc. Norm. Super. Pisa, Cl. Sci., V. Ser. 1, 187-202 (2002)

18. Jabin, P.E., Perthame, B.: Compactness in Ginzburg-Landau energy by kinetic averaging. Commun. Pure Appl. Math. 54, 1096-1109 (2001)

19. Jin, W., Kohn, R.V.: Singular perturbation and the energy of folds. J. Nonlinear Sci. 10, 355-390 (2000)

20. Kruzkov, S.N.: First order quasilinear equations in several independent variables. Math. USSR-Sbornik 10, 217-243 (1970)

21. Lecumberry, M., Rivière, T.: Regularity property for Micromagnetic configurations having zero jump energy. To appear in Calc. Var. Partial Differ. Equ.

22. Lions, P.L., Perthame, B., Tadmor, E.: A kinetic formulation of multidimensional scalar conservation laws and related questions. J. Am. Math. Soc. 7, 169-191 (1994)

23. Mattila, P.: Geometry of sets and measures in Euclidean spaces. Fractal and rectifiability. Cambridge Studies in Advanced Mathematics. Cambridge: Cambridge University Press 1995

24. Ortiz, M., Gioia, G.: The morphology and folding patterns of bucking driven thin-film blisters. J. Mech. Phys. Solids 42, 531-559 (1994) 
25. Rivière, T., Serfaty, S.: Compactness, kinetic formulation and entropies for a problem related to Micromagnetics. To appear in Commun. Partial Differ. Equations

26. Rivière, T., Serfaty, S.: Limiting domain wall energy for a problem related to micromagnetics. Commun. Pure Appl. Math. 54, 294-338 (2001) 